九州大学学術情報リポジトリ

Kyushu University Institutional Repository

Breakdown of ion-polarization-correspondence and born effective charges: Algebraic formulas of accurate polarization under field

Watanabe, Yukio

Faculty of Sciences, Kyushu University

http://hdl. handle. net/2324/4102468

出版情報: Physical Review Materials. 4 (104405), pp.104405-1-104405-11，2020-10. American Physical Society

バージョン:

権利関係 : (C)2020 American Physical Society 


\title{
Breakdown of ion-polarization-correspondence and born effective charges: Algebraic formulas of accurate polarization under field
}

\author{
Yukio Watanabe* \\ Kyushu University, Fukuoka, Japan
}

(Received 20 June 2020; accepted 11 September 2020; published 8 October 2020)

\begin{abstract}
Polarization, especially of ferroelectrics FEs, is conventionally described by ion positions, e.g., by Born effective charges, where the complete entanglement of electron polarization with that of ions is implicitly assumed. We find that such descriptions or Born effective charge polarization-type approaches break down partially in the presence of high field, owing to the partial disentanglement of electrons with ions. To overcome this, we propose a correction (non-Born effective charge polarization) that calculates both macroscopic and unit-cell-by-unit-cell total polarization accurately. The accuracy of this method is demonstrated in prototypical situations of depolarization field $E_{d}$ that exists in finite-size or inhomogeneous insulating FEs: paraelectric/FE, FE capacitors, and FE/vacuum. Here, FE/vacuum are shown to be electrically identical to encountering domains. This method provides simple algebraic formulas to calculate total polarization $P_{\mathrm{S}}$ and $E_{d}$ using conventionally estimated polarizations that are obtained from local ion positions. Therefore, it can be easily used in experimental estimations of $P_{\mathrm{S}}$ and $E_{d}$, including 3D cases. For example, this method reveals that $P_{\mathrm{S}}$ varies across ferroelectric/insulator far less than the conventional estimate, which explains substantially reduced $E_{d}$ and the absence of metallicity. In addition, vortexlike domains are discussed in view of $E_{d}$. The partial disentanglement of ion and electron polarization would imply limitation of Ginzburg-Landau framework of ferroelectrics under high field.
\end{abstract}

DOI: 10.1103/PhysRevMaterials.4.104405

\section{INTRODUCTION}

Ferroelectrics (FEs) have reversible spontaneous polarization $P_{\mathrm{S}}$ that is useful in numerous applications, for which a good insulator with a wide bandgap $E g$ is desired to apply external electric field $E_{\text {ext }}$. In real, i.e., finite-size FE, the depolarization electric field $E_{d}$ exists in FEs even for $E_{\mathrm{ext}}=$ $0 ; E_{d}$ emerges by the existence of surfaces, interfaces, and inhomogeneity in the direction of $P_{\mathrm{S}}$ [Figs. 1(a)-1(c)]. $E_{d}$ is considered essential for domain configurations [1-6], and the properties and existence of FEs [7-10]. For example, a simple Kittel model based on $E_{d}$ [1] shows nanometer-scale vortexlike $P_{\mathrm{S}}$ patterns and nanodomains [6].

The emergence of the spontaneous polarization in standard FEs is described by the disappearance of inversion-symmetric ion positions. Accordingly, the phase transitions and properties of FEs are explained by the polar distortions of ion positions $u$ [2,11-14] and their dynamics, i.e., polar softmode phonons. That is, the polar distortions of ion positions are equated with the spontaneous polarization, as shown by Ginzburg-Landau theory as a linear relationship between polarization and lattice strain $\eta$, e.g., $\eta=Q P_{\mathrm{S}}^{2}$ for stress-free case, where $Q$ is constant [15] [Fig. 1(d)].

For the understanding of FEs, especially nanometer-scale FEs, atomic-scale understanding of polarization and $E_{d}$ is indispensable. Standard experimental atomic-scale estimations of spontaneous polarization are based on local ion positions or distortion $u[11,12]$ measured by x-ray diffraction or trans-

\footnotetext{
*watanabe@phys.kyushu-u.ac.jp
}

mission electron microscopy (TEM). These estimations are performed also for $E_{d} \neq 0$ and $E_{\text {ext }} \neq 0[2-5,7,8]$ and basically the same as those using phonon modes with Born effective charges $Z^{*}$ [13] that are defined at macroscopic electric field $E=0$.

These local ion-position based estimations are used also in $a b$ initio calculations; for example, the surface of freestanding FEs and head-to-head/tail-to-tail (HH-TT) domains possess metallic bands owing to $E_{d}$, when $P_{\mathrm{S}} \perp$ surfaces or $P_{\mathrm{S}} \perp$ boundaries [10,16-19]. In these cases, empirical formulas based on ion positions or Born effective charges are used [10,19].

The present paper suggests the polarization that has been missing in these conventional estimates. In case of partly metallic FE slabs [Fig. 1(e)], $E_{d}$ originates from surfaces or domain boundaries, which are absent in the extracted (copied) bulk unit-cell [Fig. 1(f)] that has the same ion positions of the unit-cell in the slab [Fig. 1(e)] (Table I). Consequently, $E_{d}$ is absent in the copied bulk unit-cell, although the ion positions are exactly the same as those in the slab and contain the ion distortion $u$ due to $E_{d}$. Because electrons move with ions, the polarization of electrons in the slab is partly included in the polarization of this extracted bulk unit-cell $P_{\mathrm{S}}{ }^{\text {iso-bulk }}$, whereas the polarization of ions in the slab is completely included in $P_{\mathrm{S}}{ }^{\text {iso-bulk }} . P_{\mathrm{S}}{ }^{\text {iso-bulk }}$ corresponds to the polarization described by conventional estimates based on local ion-positions such as Born effective charge $\Sigma Z^{*} u$ [distortion $u$ depends on $E$ : $u(E)]$.

Because of the similarity of the physics or spirit, we relate $P_{\mathrm{S}}$ iso-bulk with Born effective charges, although $P_{\mathrm{S}}$ by Born 
(a)

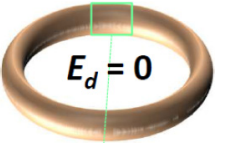

(b)

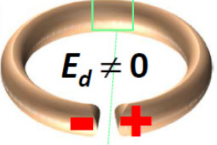

(e)
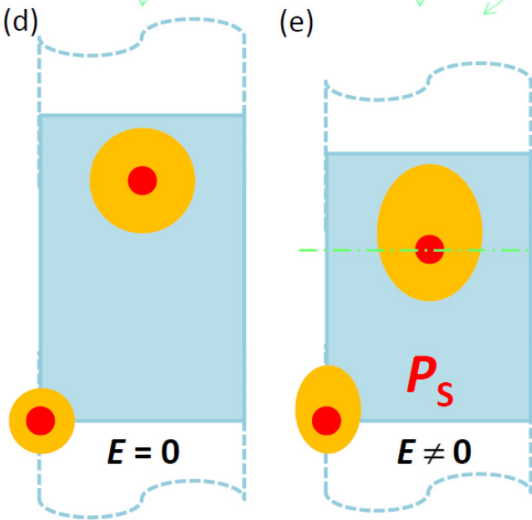

(c)

$E_{d} \neq 0$

(f)

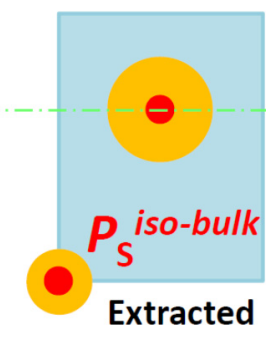

FIG. 1. Images of bulk and real FEs [(a)-(c)], the corresponding unit-cells [(d)-(f)] explaining extracted bulk unit-cell, $P_{\mathrm{S}}{ }^{\text {iso-bulk }}$ and $P_{\mathrm{el}}^{\text {Exra }}$. (a) bulk, i.e., infinite-size FE. (b), (c) real, i.e., finite-size FEs: FE/vacuum (b) and FE heterostructures (c), where $E_{d}$ emerges owing to the imperfect screening of polarization charges. (d)-(f) FE unit-cells showing ions (red) and electron clouds (orange): (d) short-circuit slab, (e) open-circuit slab, and (f) bulk having the same ion positions as (e).

effective charges may deviate from a Berry phase $P_{\mathrm{S}}$ even for $E=0$ [20] and, hence, from $P_{\mathrm{S}}$ iso-bulk. However, $P_{\mathrm{S}}$ estimated by an optimized $Z^{*}(u) u$ could agree almost perfectly with Berry phase $P_{\mathrm{S}}$ for a wide range, while the difference between $P_{\mathrm{S}}$ 's estimated with Berry phase using different exchange correlation functionals was visible (Appendix). Therefore, in general, ideal Born effective charges would be able to agree with the exact $P_{\mathrm{S}}$ by optimizing $Z^{*}$ for each specific $F E$ and including the exact dependence of $Z^{*}$ on $u$, stress $\sigma$, and specific atomic environments such as defects. To express the physics, $P_{\mathrm{S}}{ }^{\text {iso-bulk }}$ is identified with Born polarization estimated by such ideal Born effective charges $\left(P_{\mathrm{S}}^{\text {iso-bulk }}{ }_{\text {BornI }}\right)$ that are optimized for a specific FE; $P_{\mathrm{S}}^{\text {iso-bulk }}$ Born agrees perfectly with $P_{\mathrm{S}}$ estimated with ideal Berry phase $P_{\mathrm{S}}^{\text {iso-bulk }}$ Berry .

Nonetheless, $P_{\mathrm{S}}$ iso-bulk or $P_{\mathrm{S}}^{\text {iso-bulk }}{ }_{\text {BornI }}$ is considered to overlook the possibility that electrons move partly freely from ions; even when ion positions are the same, the polarization

of electrons or atomic polarization [21] may be different for $E=0$ and $E \neq 0$ [Figs. 1(e) and 1(f)]. Hence, the total polarization for $E \neq 0$ is considered as $P_{\mathrm{S}}^{\text {iso-bulk }}+P_{\mathrm{el}}^{\text {Extra }}$, where $P_{\mathrm{S}}{ }^{\text {iso-bulk }}(u)$ is the polarization of ion and electrons for $E=0$ at $u=u(E)(u$ is attained by $E)$ and $P_{\mathrm{el}}^{\text {Extra }}=\left(\varepsilon_{\text {Extra }}^{\mathrm{el}}-1\right) \varepsilon_{0} E$ is an extra polarization by $E$ ( $\varepsilon_{\mathrm{el}}^{\text {Extra }}$ : permittivity of electrons for extra polarization). The validity of these postulates shall be explained more in Sec. III and proved in Sec. IV.

When this inference is correct, this extra polarization $P_{\mathrm{el}}^{\mathrm{Exra}}$ cannot be expressed by the improvements of $P_{\mathrm{S}}^{\text {iso-bulk }}$ Berry or $P_{\mathrm{S}}^{\text {iso-bulk }}{ }_{\text {BornI }}$ that include several effects such as the dependence of $Z^{*}$ on $u, \sigma$, and $E$ [22] and the rescaling [19,23]; Total polarization is $P_{\mathrm{S}}^{\text {iso-bulk }}+\left(\varepsilon_{\mathrm{el}}^{\mathrm{Extra}}-1\right) \varepsilon_{0} E$, where we call $P_{\mathrm{el}}^{\text {Extra }}$ non-Born polarization considering ideal Born effective charges: $P_{\mathrm{S}}^{\text {iso-bulk }}$ BornI $=P_{\mathrm{S}}{ }^{\text {iso-bulk }}$. Consequently, $P_{\mathrm{el}}^{\text {Exra }}$ is not included in the molecular dynamics (MD) simulations based on effective Hamiltonian [9,24] and classical (non- $a b$ initio) MD simulations [25]. Similarly, the accurate experimental estimation of polarization from local ion positions or $u[2-5,7,8,11,12]$ is $P_{\mathrm{S}}$ iso-bulk , and, therefore, does not contain $P_{\mathrm{el}}^{\text {Exra }}$.

We have found that $P_{\mathrm{el}}^{\mathrm{Exra}}$ often reduces $E_{d}$ drastically and propose a method to calculate this polarization, where $\varepsilon_{\text {el }}^{\text {Extra }}$ can be sufficiently approximated by the static permittivity of electrons $\varepsilon^{\mathrm{el}}$. For paraelectric under low $E, P_{\mathrm{el}}^{\text {Exra }}$ may be corrected by rescaling $P_{\mathrm{S}}=C_{\mathrm{R}} P_{\mathrm{S}}$ iso-bulk $[19,23]$, where $C_{\mathrm{R}}=\left(1+\left(\varepsilon^{\mathrm{el}}-1\right) / \chi^{\mathrm{ion}}\right) \approx 0.90$ and $\chi^{\text {ion }}$ is the susceptibility due to ions. This formula is equivalent to rescaling of Born effective charge $Z^{* \mathrm{R}}: Z^{* \mathrm{R}}=C_{\mathrm{R}} Z^{*}$. On the contrary, the present extra polarization $P_{\mathrm{el}}^{\mathrm{Exra}}$ is based on the polarization independent of $u$ 's as expressed in the second term in $P_{\mathrm{S}}$ iso-bulk + $\left(\varepsilon_{\mathrm{el}}^{\mathrm{Extra}}-1\right) \varepsilon_{0} E$; consequently, $E_{d}$ is not determined by local $P_{\mathrm{S}}{ }^{i \mathrm{l}-\text { bulk }}$ as in the rescaling [23] but is determined by global distribution of $P_{\mathrm{S}}^{\text {iso-bulk }}$ as in the equations in Sec. III. Also $P_{\mathrm{el}}^{\mathrm{Exra}}$ is different from the $E$-dependence $\Sigma\left[Z^{*}+\Delta Z^{*}(E)\right] u$ proposed for semiconductors [22].

Using the consistencies of conventionally estimated polarization $P_{\mathrm{S}}$ iso-bulk [11-15], we propose a method that calculates $P_{\mathrm{el}}^{\text {Exra }}$ and total polarization, including three-dimensional (3D) cases. This method can also be performed only with global ion position data and, hence, is usable in experimental studies such as those using TEM $[2-5,7,8]$. We demonstrate the necessity and accuracy of non-Born polarization $P_{\mathrm{el}}^{\text {Exra }}$ in prototypical situations of $E_{d}: \mathrm{FE} / \mathrm{vacuum}$, paraelectric/FE, and

TABLE I. Lattice constants and ion positions of tetragonal $\mathrm{BaTiO}_{3}$ exemplarily explaining the procedure to obtain the extracted (copied) bulk unit-cell for $P_{\mathrm{S}}$ iso-bulk [Fig. 1(f)] that has the same ion positions of the unit-cell in the slab [Fig. 1(e)]. This example corresponds to BTO in $\mathrm{BTO}(7 \mathrm{PBE}+U) / \mathrm{STO}$ in Fig. 4, and ion positions are shown in fractional coordinates. The second row shows the lattice constants and ion positions of bulk $\mathrm{BaTiO}_{3}$. The third and fourth rows show the lattice constants and ion positions of BTO unit-cell in the center of BTO layers in the BTO/STO slab, where the fractional coordinates are with respect to the lattice constants of slab and the unit-cell, respectively. The fifth row shows the lattice constants and ion positions of BTO unit-cell of extracted unit-cell, which is identical with the fourth row.

\begin{tabular}{lccccccc}
\hline $\begin{array}{l}\text { Fractional coordinates } \\
\text { in } a \text { and } c\end{array}$ & $a(\AA)$ & $z_{\text {bottomBa }}\left(0,0, z_{\mathrm{Ba}}\right)$ & $z_{\text {topBa }}\left(0,0, z_{\mathrm{Ba}}\right)$ & $c(\AA)$ & $z_{\mathrm{Ti}}\left(0.5,0.5, z_{\mathrm{Ti}}\right)$ & $z_{\mathrm{O} 2}\left(0,0.5, z_{\mathrm{Ti}}\right)\left(0.5,0, z_{\mathrm{Ti}}\right)$ & $z_{\mathrm{O} 1}\left(0.5,0.5, z_{\mathrm{Ti}}\right)$ \\
\hline Bulk & 3.979 & 0 & 1 & 4.069 & 0.5141 & 0.4837 & -0.0301 \\
Slab center & 3.947 & 0.29749 & 0.38245 & 47.920 & 0.34118 & 0.33914 & 0.29564 \\
Slab center & 3.947 & 0 & 1 & 4.071 & 0.5142 & 0.4903 & -0.0218 \\
Copied bulk & 3.947 & 0 & 1 & 4.071 & 0.5142 & 0.4903 & -0.0218 \\
\hline \hline
\end{tabular}




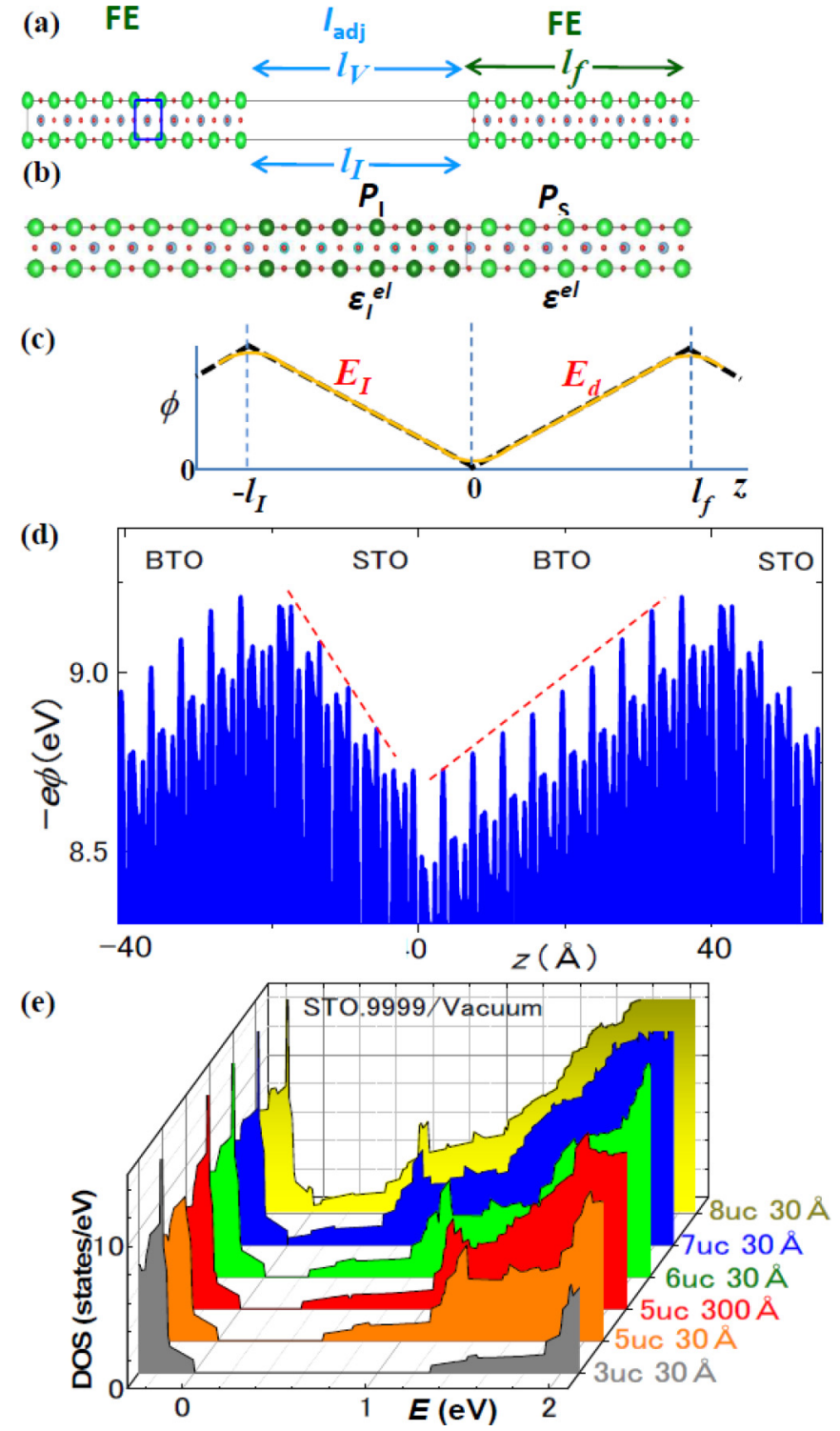

FIG. 2. Atomic model of (a) FE/vacuum and (b) FE/I $I_{\text {adj }}$ superlattice, and (c) corresponding macroscopic potential $\phi$. (d) Example of the estimation of $a b$ initio $E_{d}\left(E_{d}^{a b i n i t i o}\right)$ from the highest planar averaged atomic potential $\phi$ by the ab initio calculation of 10-unitcell BTO/5-unit-cell STO. (e) DOS of STO.9999/vacuum, where $l_{f}$ in the unit of unit-cells and $l_{V}$ in $\AA$ are shown on the right.

FE capacitors [Figs. 1(b), 1(c), and 2]. Here, FE/vacuum can represent HH-TT domains. In view of reduced $E_{d}$, the vortex domains [3-5,9,24,25] are discussed in comparison with flux closures [26-28].

\section{TECHNICAL DETAILS}

To calculate $P_{\mathrm{S}} a b$ initio, we used insulating FE slabs consisting of $\mathrm{SrTiO}_{3}$ (STO) and archetypical FE $\mathrm{BaTiO}_{3}$ (BTO). For the achievement of the insulativity of FE, which was necessary for the $a b$ initio calculation of $P_{\mathrm{S}}$ of a whole slab, FE in FE/vacuum [Fig. 2(a)] required a small $P_{\mathrm{S}}$ and a large $E g$ (Appendix). These requirements were achieved by $\mathrm{SrO}-$ terminated STO's (P4mm) having $a$-axis lattice constant $0.5 \%$ longer and $0.01 \%$ shorter than that of the theoretical cubic phase [29]. We call them STO1.005 and STO.9999, respectively, of which lattice-constants $a$ and $c$, and $P_{\mathrm{S}}$ are $3.902 \AA$, $3.896 \AA, 3.56 \mu \mathrm{C} / \mathrm{cm}^{2}, 3.897 \AA, 3.898 \AA$, and $6.15 \mu \mathrm{C} / \mathrm{cm}^{2}$, respectively [29]. For FE/vacuum, geometries were not relaxed, because otherwise FE disappears (Appendix); The FE unit-cells in each slab retained the ion positions of STO1.005 or STO.9999. Therefore, $P_{\mathrm{S}}$ iso-bulk of any unit-cell was the same as the above bulk value: $3.56 \mu \mathrm{C} / \mathrm{cm}^{2}$ or $6.15 \mu \mathrm{C} / \mathrm{cm}^{2}$. These calculations were only for the examination of $P_{\mathrm{el}}^{\text {Exra }}$ and $\varepsilon_{\mathrm{el}}^{\text {Extra }}$ and were not intended to explain experiments.

BTO/STO superlattices were used as FE/insulator heterostructures [Fig. 2(b)]. All the calculated forces were $<1 \mathrm{meV} / \AA$ after geometry relaxation. In the calculation of BTO capacitor structures with PBEsol, a standard electrode material $\mathrm{SrRuO}_{3}$ was 5 unit-cell thick $\sim 20 \AA$. The $a$-lattice constant of $\mathrm{BTO} / \mathrm{SrRuO}_{3}$ was fixed at the theoretical $a$ of cubic STO, and all other ion positions were relaxed, which corresponds to the epitaxial films on STO substrates. The surfaces of the BTO and $\mathrm{SrRuO}_{3}$ were $\mathrm{TiO}_{2}$ and $\mathrm{SrO}$, respectively.

In the remainder, $I_{\text {adj }}$ stands for both vacuum in $\mathrm{FE} /$ vacuum and insulator in FE/insulator, and $P_{I}$ and $E_{I}$ stand for the polarization and the depolarization field in $I_{\text {adj }}$, respectively [Fig. 2(a)]. $P_{\mathrm{S}}{ }^{\text {iso-bulk }}$ and $P_{I}^{\text {iso-bulk }}$ denote the polarizations of the extracted unit-cells from FE and $I_{\text {adj }}$, respectively, which can be calculated from local ion positions. An example of the extracted unit-cell is indicated by a blue small rectangle in Fig. 2(a). The permittivity's of electrons and the thickness of FE and $I_{\mathrm{adj}}$ are denoted by $\varepsilon^{\mathrm{el}}, \varepsilon_{I}^{\mathrm{el}}, l_{f}$, and $l_{I}\left(=l_{V}\right.$ for $I_{\text {adj }}=$ vacuum $)$, respectively, and the length of the slab $l_{\mathrm{SC}}$ is $l_{f}+l_{I}$. The periodic behavior of the potential in Fig. 2(c) suggests $l_{f} E_{d}=-l_{I} E_{I}$. Ab initio $E_{d}\left(E_{d}^{a b i n i t i o}\right)$ was obtained from the envelope of the peak tops of atomic potential, where the dashed red lines in Fig. 2(d) are the example of this envelope (also in Appendix).

In these calculations, no dipole correction was applied, as usual for the studies of $\mathrm{FE} / I_{\text {adj }}$ superlattices and FE capacitors (The appropriateness for FE/vacuum is explained in Appendix). These $a b$ initio calculations rigorously correspond to the macroscopic model in Fig. 2(c), which is sufficient for the present paper. The correctness of no dipole correction is evident in the excellent agreements between $E_{d}^{a b}$ initio and analytical $E_{d}$ [30] and between $a b$ initio and analytical $P_{\mathrm{S}}$ (Sec. IV). These results also explain quantitatively the decrease of $E_{d}$ with $l_{f}$ and the emergence of metallicity in Fig. 2(e).

In the $a b$ initio calculations with VASP [31], the projector augmented wave (PAW) method [32] was used with PBEsol functional [33], a Monkhorst-Pack [34] mesh of $8 \times 8 \times 2$, and with an energy cutoff of $650 \mathrm{eV}$. Ab initio $P_{\mathrm{S}}$ and $P_{\mathrm{S}}{ }^{\text {iso-bulk }}$ were obtained by Berry phase calculations [35]. In case of FE/vacuum, the dipole moment of a whole FE/vacuum slab was calculated with Berry phase, and $P_{\mathrm{S}}$ was obtained by dividing this dipole moment by the volume of FE part $\left(a \times a \times l_{f}\right)$, which we call a "rigorously calculated $P_{\mathrm{S}}$ of the slab." The calculations of BTO/STO superlattices were examined also with PBE functional [36] with Hubbard $U$ [37] $(\mathrm{PBE}+U: U$ on Ti $3 \mathrm{~d}$ and $\mathrm{O} 2 \mathrm{p})$. Optical permittivity of bulk STO and BTO was calculated with ex- 


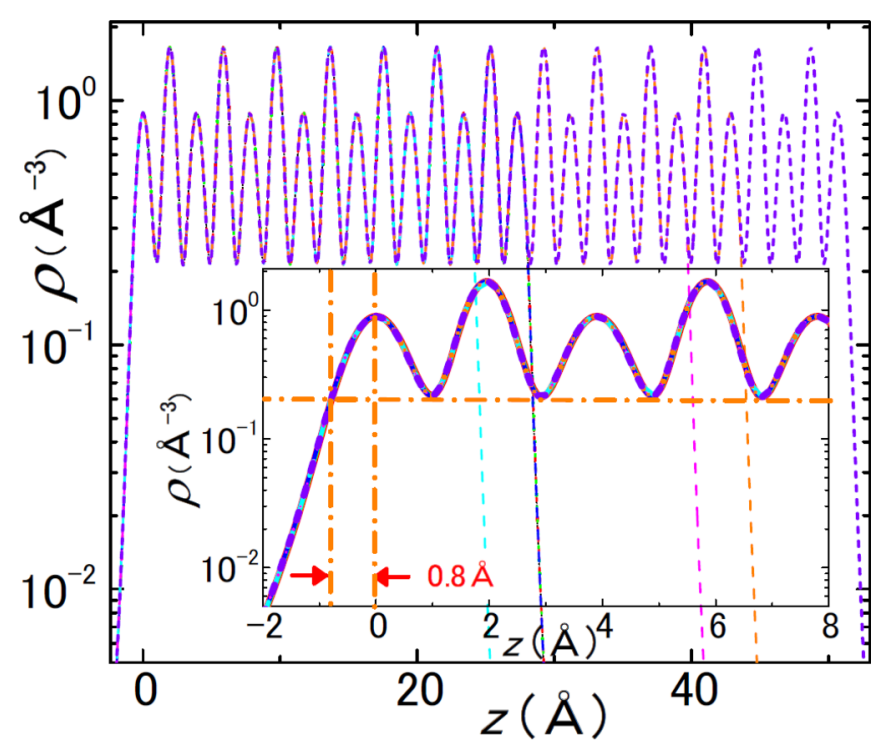

FIG. 3. Planar averaged electron density $\rho$ for estimating the FE thickness $l_{f}$. All the eight $\rho$ 's behave the same near the surface, supporting the use of the same smearing length $\sim 0.8 \AA$ for different slabs. Inset shows the enlarged view near the surface.

act diagonalization and random phase approximation (RPA). $E$ g's are calculated with PBEsol, $\mathrm{PBE}+U$, and a hybrid functional HSEsol [38], where PBEsol is known to underestimate $E$ g's typically by $30 \%$, while HSEsol yields appropriate values [39].

In the present calculations of $\mathrm{FE} /$ vacuum, it is important how to define the location of FE surface, i.e., $l_{f}$. Therefore, $l_{f}$ was estimated from the planer averaged electron density $\rho$ profiles in Fig. 3, which displays that all the $\rho$ profiles of 8 different slabs exhibit the same behaviors near the surface. This is more evident in the enlarged view at $z \approx 0$, where all the $\rho$ profiles of eight different slabs overlap. The $\rho$ peaks at the right and left end $(z=0)$ correspond to the $\mathrm{BaO}$ of the top and bottom surface, respectively. $\rho$ at $z=-0.8 \AA$ is same as the minimum $\rho$ of inner part in all the $\rho-z$ curves. This means that the region of $z=0 \sim-0.8 \AA$ should be considered as a part of $\mathrm{FE}$, and $l_{f}$ is the distance of top and bottom outermost ion (Ba) position plus $2 \times 0.8 \AA$. As for $l_{I}, l_{I}=l_{\mathrm{SC}}-l_{f}$.

In the calculation of BTO/STO, $l_{f}$ was defined as the distance between the top and bottom Ti ions of BTO, and $l_{I}=l_{\mathrm{SC}}-l_{f}$. In the calculation of $\mathrm{BTO} / \mathrm{SrRuO}_{3}$ capacitor structures, the effective $l_{f}\left(l_{f}^{\text {eff }}\right)$ was $l_{\mathrm{T}-\mathrm{B}}-$ uc $\mathrm{BTO}$, where $l_{\mathrm{T}-\mathrm{B}}$ is the distance between top and bottom $\mathrm{Ti}$ (top and bottom of BTO are $\mathrm{TiO}_{2}$ 's) and $\mathrm{uc}_{\text {вто }}$ is the length of a BTO unitcell. The results with $l_{f}^{\text {eff }}=l_{\mathrm{T}-\mathrm{B}}-1.5 \mathrm{uc}_{\mathrm{BTO}}$ were almost the same.

\section{METHODS}

Section I postulated $P_{\mathrm{S}}=P_{\mathrm{S}}{ }^{\text {iso-bulk }}+P_{\mathrm{el}}^{\text {Exra }}$ with $P_{\mathrm{el}}^{\text {Exra }}=$ $\left(\varepsilon_{\mathrm{el}}^{\mathrm{Extra}}-1\right) \varepsilon_{0} E$, of which technical details are explained in Fig. 1, Table I, and the Appendix. The background of the formulas are as follows. Within Born-Oppenheimer adiabatic approximation, electron distribution $\rho$ is considered to depend on a total field: atomic fields from ions $\left(E_{\text {ion }}^{a}\right)$, of which macroscopic average is zero, and macroscopic field $E$, e.g., $E_{d} . E$ displaces ions $(u(E))$, yielding ion polarization $P_{\text {ion }}(u)$. Because of the coupling to $E_{\text {ion }}^{a}(u)$ and $E$, $\rho\left(E_{\text {ion }}^{a}(u), E\right)=\rho(u, E)$, which yields electron polarization $P_{e}(\rho)=P_{e}(\rho(u, E))=P_{e}(u, E)$. This expression $P_{e}(u, E)$ is needed, because in general cases electrons and each ion are governed by their own different mechanics.

For a weak $E, P_{\mathrm{S}}=P_{\text {ion }}(u)+P_{e}(u, 0)$ can be rigorously expressed by ideal Born effective charges $P_{\mathrm{S}}=\Sigma Z^{*} u$, thanks to the robust interactions between ions and electrons $\left(E_{\mathrm{ion}}^{a}\right)$. As the last paragraph indicates, such complete entanglement of $P_{e}$ with $u$ and, hence, ion polarization $P_{\text {ion }}$ is approximate, when $E$ is nonnegligible in comparison with $E_{\text {ion }}^{a}$. That is, the straightforward expression $P_{\mathrm{S}}=P_{\text {ion }}(u)+P_{e}(\rho)$ is more appropriate than $P_{\mathrm{S}}=\Sigma Z^{*} u$.

Therefore, $\quad P_{\mathrm{S}}=P_{\text {ion }}(u)+P_{e}(\rho)=P_{\text {ion }}(u)+P_{e}(u, E) \approx$ $P_{\text {ion }}(u)+P_{e}(u, 0)+\Delta P_{e} E=P_{\mathrm{S}}$ iso-bulk $+P_{\mathrm{el}}^{\text {Exra }}, \quad$ where $P_{\mathrm{S}}^{\text {iso-bulk }} \equiv P_{\text {ion }}(u(E))+P_{e}(u(E), 0), \quad P_{\mathrm{el}}^{\mathrm{Exra}} \equiv \Delta P_{e} E, \quad$ and quasi-equality is for $E \ll E_{\text {ion. }}^{a}$. Below, $\varepsilon_{\mathrm{el}}^{\text {Extra's }}$ are approximated by the static permittivity of electrons $\varepsilon^{\mathrm{el}}$, as explained by the end of Sec. III.

The 1D-polarizations below denote the components perpendicular to the surfaces and interfaces (1D refers to the case where properties change only along one coordinate as in Fig. 2). Therefore, the polarizations below such as $P_{\mathrm{S}}$, $P_{I}, P_{\mathrm{S}}$ iso-bulk , and $P_{I}^{\text {iso-bulk }}$ should be multiplied by $\cos \theta(\theta$ : incidence angle), when the total polarizations are oblique to the surfaces and interfaces (Appendix).

\section{A. Basic equations for 1D $N$-insulators and layer-by-layer}

In the macroscopic derivation of $E_{d}, E_{d}$ is constant except for surfaces and boundaries, which is confirmed in Fig. 2(d) and all the potential profiles studied. The thickness of surface layers or boundaries, in which the potential corresponding to macroscopic $E_{d}$ deviates from atomic potential, is only $1 \sim 2$ unit-cell. In case of FE/vacuum, this deviation in 1-unit-cell thick regions is mainly due to work function (Appendix).

The other surface effects not included in the macroscopic derivation of $E_{d}$ are buckling, i.e., inward dipole layer at the surface with $\sim 1$ unit-cell thickness [40] and the atomic-scale variations of polarization $P$ at the surface. However, these effects do not change the estimate of macroscopic $E_{d}$ either, while we need to change the definition of the effective $l_{f}$ as the thickness of the inner layers having a constant polarization $P$; Exemplarily in case of freestanding insulating 1D-FE, $\varepsilon_{0} E_{d}+P=0$ shows $E_{d}(z)=-P(z) / \varepsilon_{0}$, which means that $E_{d}$ is unaffected by other locations as long as an end of FE is in an open vacuum. Consequently, we could confirm that the macroscopic formula of $E_{d}$ agreed excellently with the ab initio $E_{d}\left(E_{d}^{a b}\right.$ initio $)$ in all the a-few-nanometer-scale FEs studied here.

According to Sec. I, the correction to $P_{\mathrm{S}}{ }^{\text {iso-bulk }}$ is given by

$$
P s=P s^{\text {iso-bulk }}+\left(\varepsilon^{e l}-1\right) \varepsilon_{0} E_{d} .
$$


In Eq. (1), $\varepsilon_{\mathrm{el}}^{\text {Extra }}$ is approximated by $\varepsilon^{\mathrm{el}}$ and $E_{d}$ is given by ab initio calculation [Fig. 2(d)] or by

$$
E_{d}=-\frac{P_{S}^{\text {iso-bulk }}-P_{I}^{\text {iso-bulk }}}{\varepsilon_{0}\left(\varepsilon^{e l}+\frac{l_{f}}{l_{I}} \varepsilon_{I}^{e l}\right)},
$$

for a 1D system consisting of two insulators [Figs. 2(a) and 2(b)]. Equation (2a) is derived from Eq. (2b) for $N=2$. Similarly, $P_{I}=P_{I}^{\text {iso-bulk }}+\left(\varepsilon_{I}^{\mathrm{el}}-1\right) \varepsilon_{0} E_{I}$, where $E_{I}$ is given by $a b$ initio calculation [Fig. 2(d)] or $E_{I}=-E_{d} l_{f} / l_{I}$. The examples corresponding to Eq. (2a) are FE/vacuum, FE/insulator, and HH-TT domains [18]. For vacuum, $P_{I}^{\text {iso-bulk }}=0$ and $\varepsilon_{I}^{\mathrm{el}}=1$, yielding $P_{\mathrm{S}}=P_{\mathrm{S}}{ }^{\text {iso-bulk }}\left\{1-\left(\varepsilon^{\mathrm{el}}-1\right) /\left(\varepsilon^{\mathrm{el}}+l_{f} / l_{I}\right)\right\}$.

Polarization and $E_{d}$ of the $k$ th layer in a $1 \mathrm{D}$ system consisting of $N$ insulating layers are obtained by using Eq. (1): $P_{j}=$ $P_{j}^{\text {iso-bulk }}+\left(\varepsilon_{j}^{\mathrm{el}}-1\right) \varepsilon_{0} E_{d j}\left(P_{j}\right.$ : corrected polarization at $j=$ $1 \sim N)$, the continuity of flux $P_{j}^{\text {iso-bulk }}+\varepsilon_{j}^{\mathrm{el}} \varepsilon_{0} E_{d j}=P_{j+1}^{\text {iso-bulk }}+$ $\varepsilon_{j+1}^{\mathrm{el}} \varepsilon_{0} E_{d j+1}(j=1 \sim N-1)$, and the continuity of potential for a periodic boundary condition $\Sigma_{1}^{N} l_{j} E_{d j}=0$ with $E_{d N+1}=$ $E_{d 1}, \varepsilon_{N+1}^{\text {el }}=\varepsilon_{1}^{e l}$, and $P_{N+1}^{\text {iso-bulk }}=P_{1}{ }^{\text {iso-bulk }}$. Here, $P_{j}, P_{j}^{\text {iso-bulk }}$, $\varepsilon_{j}^{\mathrm{el}}, E_{d j}$, and $l_{j}$ are the corrected (total) polarization, the polarization of an extracted bulk unit-cell, the permittivity of electrons, the depolarization field, and the thickness of the $j$ th insulating layer. These relations yield

$$
E_{d k}=-\frac{\sum_{j=1}^{N}\left\{\left(P_{k}^{\text {iso-bulk }}-P_{j}^{\text {iso-bulk }}\right) l_{j} / \varepsilon_{j}^{e l}\right\}}{\varepsilon_{k}^{e l} \varepsilon_{0} \sum_{j=1}^{N} \frac{l_{j}}{\varepsilon_{j}^{e l}}}(k=1 \sim N),
$$

which gives $P_{k}$ by Eq. (1). The periodic boundary condition of electrostatic potential is clearly satisfied in short circuit 1D systems and also in open circuit 1D systems; For example, a freestanding $\mathrm{FE}$ in open vacuum is expressed by a periodic system [Fig. 2(a)] with $l_{V}=\infty$.

Equation (2b) for $N=2$ yields Eq. (2a), by $E_{d 1}=E_{d}$, $P_{1}{ }^{\text {iso-bulk }}=P_{\mathrm{S}}{ }^{\text {iso-bulk }}, \varepsilon_{1}^{\mathrm{el}}=\varepsilon^{\mathrm{el}}, l_{1}=l_{f}, P_{2}{ }^{\text {iso-bulk }}=P_{I}{ }^{\text {iso-bulk }}$, $\varepsilon_{2}^{\mathrm{el}}=\varepsilon_{I}^{\mathrm{el}}$, and $l_{2}=l_{I}$. The conducting layer, in which $\phi$ is constant, is considered as none and expressed by $\varepsilon_{j}^{\mathrm{el}}=\infty$ or $l_{j}=0$; an example is an inner part of an electrode metal.

$P_{j}^{\text {iso-bulk }}$ of some insulating layers such as the screening layers of metal electrodes is difficult to measure or define and usually expressed by $P_{j}=\left(\varepsilon_{j}-1\right) \varepsilon_{0} E_{d j}$. When a $1 \mathrm{D}$ system consists of such layers and the layers expressed by $P_{j}=P_{j}^{\text {iso-bulk }}+\left(\varepsilon_{j}^{\mathrm{el}}-1\right) \varepsilon_{0} E_{d j}$ in the $k^{\text {th }}$ insulating layer having $\varepsilon_{k}^{\mathrm{el}}$ is

$$
E_{d k}=-\frac{\sum_{\left(\varepsilon_{j}^{e l}\right)}\left\{\left(P_{k}^{\text {iso-bulk }}-P_{j}^{\text {iso-bulk }}\right) l_{j} / \varepsilon_{j}^{e l}\right\}+\sum_{\left(\varepsilon_{j}\right)}\left\{\frac{P_{k}^{\text {iso-bulk }} l_{j}}{\varepsilon_{j}}\right\}}{\varepsilon_{k}^{e l} \varepsilon_{0}\left(\sum_{\left(\varepsilon_{j}^{e l}\right)} \frac{l_{j}}{\varepsilon_{j}^{e l}}+\sum_{\left(\varepsilon_{j}\right)} \frac{l_{j}}{\varepsilon_{j}}\right)}
$$

where $\Sigma_{\left(\varepsilon_{j}^{\mathrm{el}}\right)}$ and $\Sigma_{\left(\varepsilon_{j}\right)}$ are sum over the insulating layers expressed by $P_{j}=P_{j}^{\text {iso-bulk }}+\left(\varepsilon_{j}^{\mathrm{el}}-1\right) \varepsilon_{0} E_{d j}$ and $P_{j}=\left(\varepsilon_{j}-\right.$ 1) $\varepsilon_{0} E_{d j}$, respectively.

Unit-cell-scale layer-by-layer $P_{j}$ and $E_{d}$ (1D): Layer-bylayer polarization (corrected) $P_{j}$ and $E_{d}$ is obtained from ion positions in each unit-cell [Fig. 4(a)]. By regarding $P_{j}^{\text {iso-bulk }}$ and $l_{j}$ as $P_{\mathrm{S}}$ iso-bulk and $c$-lattice constant of each unit-cell, respectively, Eqs. (1) and (2b) [or (2c)] provide layer-layer $P_{\mathrm{S}}$ and $E_{d}$ of each unit-cell [Fig. 4(b)]. In Eqs. (1), (2b), and $(2 \mathrm{c}), P_{j}^{\text {iso-bulk }}$ and $l_{j}$ can be approximated by average or typical $P_{j}^{\text {iso-bulk }}$ and $l_{j} . \varepsilon_{j}^{\mathrm{el}}$ and $\varepsilon_{j}$ can also be approximated as the constant for the same material with the same symmetry (Fig. 5). Because electronic peculiarity at the surface and metallic interface is not considered, this estimate may be inaccurate for the unit-cells at these locations.

\section{B. Equations for typical situations}

Freestanding $\mathrm{FE}$ and $\mathrm{HH}$-TT domains: For freestanding $\mathrm{FE}$ in vacuum, Eq. (2b) yields, $E_{d}=-P_{\mathrm{S}}{ }^{\text {iso-bulk }} / \varepsilon_{0} \varepsilon^{\mathrm{el}}$ for $N=2$, $E_{d 1}=E_{d}, P_{1}{ }^{\text {iso-bulk }}=P_{\mathrm{S}}{ }^{\text {iso-bulk }}, \varepsilon_{1}^{\mathrm{el}}=\varepsilon^{\mathrm{el}}, P_{2}{ }^{\text {iso-bulk }}=0, \varepsilon_{2}^{\mathrm{el}}=$ 1 , and $l_{2}=\infty$. This formula $E_{d}=-P_{\mathrm{S}}{ }^{\text {iso-bulk }} / \varepsilon_{0} \varepsilon^{\mathrm{el}}$ is the same for periodic HH-TT domains as given by Eq. (2b) with $N=2, \quad E_{d 1}=E_{d}, \quad-P_{2}{ }^{\text {iso-bulk }}=P_{1}{ }^{\text {iso-bulk }}=P_{\mathrm{S}}{ }^{\text {iso-bulk }}, \varepsilon_{2}^{\text {el }}=$ $\varepsilon_{1}^{\mathrm{el}}=\varepsilon^{\mathrm{el}}$, and $l_{2}=l_{1}=l_{f}$.

Capacitors: Another common situation is a $\mathrm{FE}(j=2)$ sandwiched with metals or semiconductors $(j=1,3)$ in a short-circuited condition $(N=3)$. In this case, the effect of these metals and semiconductors appears only through a fi- nite screening length. Equation (2c) is applicable with $P_{j}=$ $\left(\varepsilon_{j}-1\right) \varepsilon_{0} E_{d j}(j=1,3)$ and $\Sigma_{\left(\varepsilon_{j} e l\right)}$ in the numerator being zero. By setting $P_{2}{ }^{\text {iso-bulk }}=P_{\mathrm{S}}{ }^{\text {iso-bulk }}$ in $\Sigma_{\left(\varepsilon_{j}\right)}$ of the numerator, and $\varepsilon_{2}^{\mathrm{el}}=\varepsilon^{\mathrm{el}}$ and $l_{2}=l_{f}$ in $\Sigma_{\left(\varepsilon_{j} e l\right)}$ of the denominator,

$E_{d}=E_{d 2}=-\left(P_{\mathrm{S}}{ }^{\text {iso-bulk }} l_{1} / \varepsilon_{1}+P_{\mathrm{S}}{ }^{\text {iso-bulk }} l_{3} / \varepsilon_{3}\right) / \varepsilon^{\mathrm{el}} \varepsilon_{0}$ $\left(l_{2} / \varepsilon^{\mathrm{el}}+l_{1} / \varepsilon_{1}+l_{3} / \varepsilon_{3}\right)=-P_{\mathrm{S}}{ }^{\text {iso-bulk }} / \varepsilon_{0}\left(\varepsilon^{\mathrm{el}}+\varepsilon_{1} l_{f} / 2 l_{1}\right)$. The last equality is for identical electrode materials: $\varepsilon_{1}=\varepsilon_{3}$ and $l_{1}=l_{3}$, which is similar to a well-known formula [41]. This $E_{d}$ yields $P_{\mathrm{S}}=\left\{1-\left(\varepsilon^{\mathrm{el}}-1\right) /\left(\varepsilon^{\mathrm{el}}+\varepsilon_{1} l_{f} / 2 l_{1}\right)\right\} P_{\mathrm{S}}{ }^{\text {iso-bulk }}$.

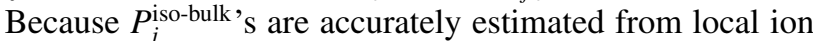
positions by either the ab initio calculation or empirical formula [12] of an extracted unit-cell, these equations show that the corrected $P_{\mathrm{S}}\left(P_{j}\right)$ and $E_{d}\left(E_{d j}\right)$ are obtainable from global ion positions. To obtain a macroscopic $E_{d}$ (not layer by layer $\left.E_{d}\right), P_{\mathrm{S}}{ }^{\text {iso-bulk }}$ and $P_{I}{ }^{\text {iso-bulk }}\left(P_{j}^{\text {iso-bulk }}\right)$ can be approximated as constant except for the 1 unit-cell at the interface [Fig. 4(a)].

Metallic surface and domain boundaries: $E_{d}^{a b}$ initio is obtainable from $a b$ initio potential profiles [Fig. 2(d)]. This estimation is possible, even when the surface and domain boundaries exhibit metallic energy band [16-18]. $E_{d}$ is estimated experimentally as $E_{d}=E g / e l_{f}^{\text {insulate }}$, where $l_{f}^{\text {insulate }} \equiv$ $l_{f}-2 l_{e h}$ and $l_{e h}$ is the thickness of metallic layer and $e$ is elementary charge. $l_{e h}$ is $2 \AA \sim 10 \AA$. Therefore, the corrected $P_{\mathrm{S}}\left(P_{j}\right)$ and $E_{d}\left(E_{d j}\right)$ are also obtainable from theoretical or experimental global ion positions.

When $l_{f}^{\text {insulate }}$ of FE is too short, the $a b$ initio potential profile of $I_{\text {adj }}$ is used to calculate $E_{d}$ through $E_{d}=-l_{I} E_{I} / l_{f}^{\text {insulate }}$ . Preferentially, the method of the estimation of $E g$ is same as that used for $P_{\mathrm{S}}$ iso-bulk , When $P_{\mathrm{S}}{ }^{\text {iso-bulk }}$ is calculated $a b$ initio 


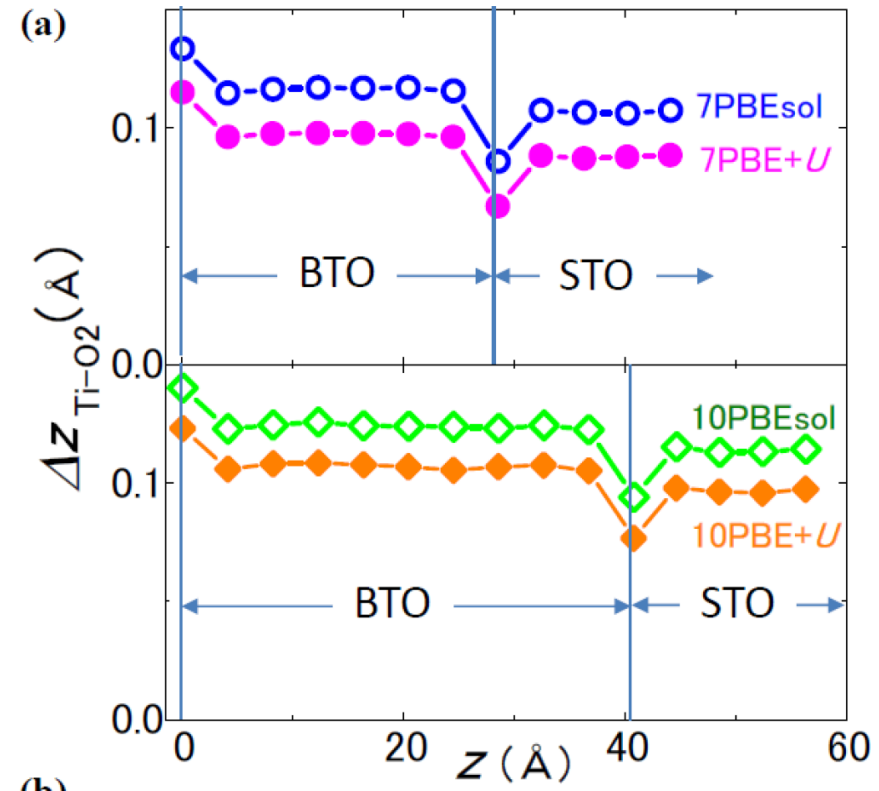

(b)

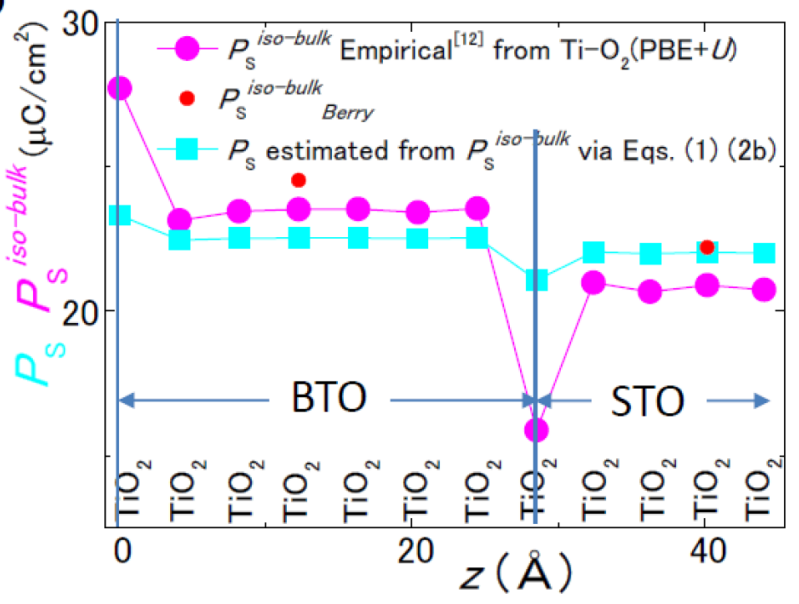

FIG. 4. (a) Layer-by-layer plot of the distance between Ti ion and $\mathrm{O}$ ion in $\mathrm{TiO}_{2}$ plane along $c$-axis of $n$-unit-cell BTO/5-unit-cell STO superlattices $(n=7,10)$. (b) $P_{\mathrm{S}}{ }^{\text {iso-bulk }}$ of each unit-cell having the ion positions shown by filled pink circles in (a) is represented by filled pink circles in (b). The corresponding $P_{\mathrm{S}}^{\text {iso-bulk }}{ }_{\text {Berry }}$ is shown by a small red circle. The corrected polarization $P_{\mathrm{S}}$ is shown by filled light-blue squares.

with PBEsol, $E g$ should be calculated with PBEsol, and when $P_{\mathrm{S}}$ iso-bulk is experimental, $E g$ should be experimental.

Defects and impurities: In the above procedures, Eqs. (2a)(2c) and the ab initio calculations assume that the effects of defects and impurities are negligible. When the effects of defects and impurities are so evident that $E_{d} \approx 0$, the present method is unnecessary.

\section{3D $P_{\mathrm{S}}$ and $E_{d}$ and representative value}

Equations (1) and (2b) or (2c) can provide 3D distributions of $P_{j}$ and $E_{d}$ by changing $P_{j}^{\text {iso-bulk }}, E_{d j}$, and $l_{j}$ to vector components $P_{\alpha j x j y j z}^{\mathrm{iso}}, E_{d \alpha j x j y j z}, l_{\alpha j x j y j z}$, and $\varepsilon_{\alpha j x j y j z}^{\mathrm{el}}$, respectively, where $\alpha=x, y, z$, and $j_{x}=1 \sim N_{x}, j_{y}=1 \sim N_{y}$, and $j_{z}=1 \sim N_{z}$ denote the center of the uniform $P_{\mathrm{S}}{ }^{\text {iso-bulk }}$ block in Cartesian coordinates. In a unit-cell scale representation,

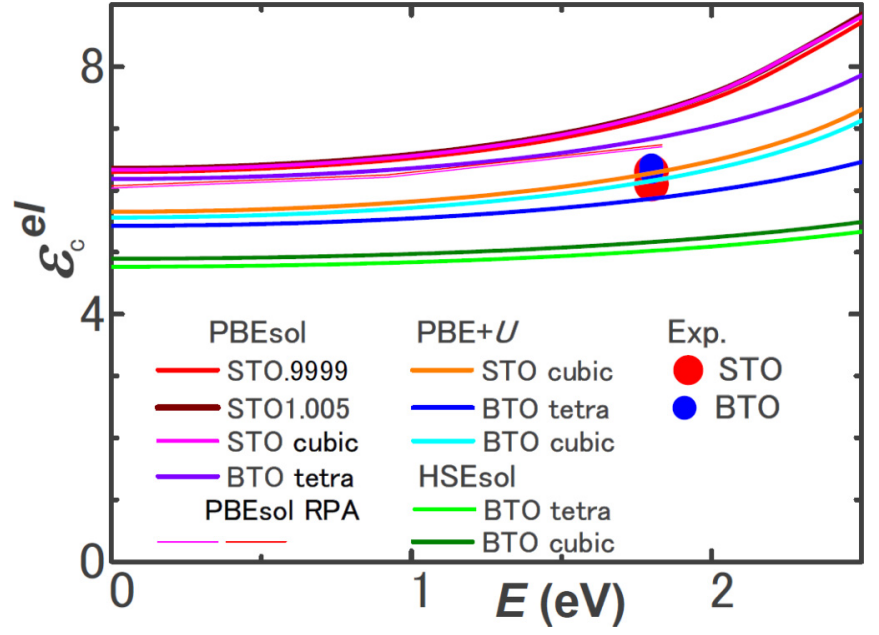

FIG. 5. Permittivity of electrons $\varepsilon^{\mathrm{el}}$ along $c$ axis of STO1.005, STO.9999, cubic STO and BTO by $a b$ initio calculations with PBEsol, $\mathrm{PBE}+U$, and a hybrid functional HSEsol, while $\varepsilon^{\mathrm{el}}$ of STO1.005 is overlapped with that of cubic STO and almost invisible. Experimental optical $\varepsilon^{\mathrm{el}}$ 's [42] are also shown. In the PBEsol calculations of STO and STO.9999, the agreements with experiment were improved by RPA, which is shown by thin red and scarlet lines that are terminated near $1.8 \mathrm{eV}$. $\varepsilon^{\mathrm{el}}$ along $a$ axis by each functional is very close to $\varepsilon^{\mathrm{el}}$ along $c$ axis by each functional.

$j_{x}, j_{y}$, and $j_{z}$ denote the center position of a unit-cell, and $\varepsilon_{\alpha j x j y j z}^{\mathrm{el}}$ can be approximated by a constant $\varepsilon^{\mathrm{el}}$ of a given polarization direction. For example, $P_{\alpha k x k y k z}=P_{\alpha k x k y k z}^{\text {iso }}+$ $\left(\varepsilon_{\alpha k x k y k z}^{\mathrm{el}}-1\right) \varepsilon_{0} E_{d \alpha k x k y k z}$ and $E_{d z k x k y k z}=-\left\{\Sigma_{j z=1}^{N z}\left(P_{z k x k y k z}^{\text {iso }}-\right.\right.$ $\left.\left.P_{\text {zkxkyjz }}^{\text {iso }}\right) l_{z k x k y j z} / \varepsilon_{z k x k y j z}^{\mathrm{el}}\right\} /\left\{\varepsilon_{z k x k y k z}^{\mathrm{el}} \varepsilon_{0} \Sigma_{j z=1}^{N z} l_{z k x k y j z} / \varepsilon_{z k x k y j z}^{\mathrm{el}}\right\}$ for the $z$ component at the location center $\left(k_{x}, k_{y}, k_{z}\right)$ by Eq. (2b).

In the application of the formula for $2 \mathrm{D}$ and $3 \mathrm{D}$, all the boundaries should be consistently oriented; For example, the boundary between $P_{\alpha j x j y j z}^{\text {iso }}$ and $P_{\alpha j x j y j z+1}^{\text {iso }}$ should be perpendicular to $z$ axis, and the boundary between $P_{\alpha j x j y j z}^{\text {iso }}$ and $P_{\alpha j x+1 j y j z}^{\text {iso }}$ should be perpendicular to $x$ axis. Therefore, the boundaries of 2D cases form steplike shapes. Practically, even for unitcell scale $P_{j}$ and $E_{d}$ distribution, representative values of a large homogeneous block can approximate $P_{i \alpha j x j y j z}, E_{d \alpha j x j y j z}$, $l_{\alpha j x j y j z}$, and $\varepsilon_{\alpha j x j y j z}^{\mathrm{el}}$ in the above sums, except for quantities of $\left(k_{x}, k_{y}, k_{z}\right)$, i.e., the location to be calculated.

\section{Value of $\varepsilon_{\mathrm{el}}^{\text {Extra }}$}

$A b$ initio and experimental electron's linear permittivity along $c$ axis are shown in Fig. 5 [42]. The perturbation series expression of the electron's static permittivity is

$$
\begin{aligned}
\varepsilon^{e l}= & 1+2 e^{2}\left\{\sum \frac{\left|x_{u o}\right|^{2}}{U_{u}-U_{o}}+e E \sum \sum \frac{x_{o i} x_{i o} x_{o o}}{\left(U_{i}-U_{o}\right)^{2}}\right. \\
& \left.-\frac{x_{o i} x_{i u} x_{u o}}{\left(U_{i}-U_{o}\right)\left(U_{u}-U_{o}\right)}+e^{2} E^{2} . .\right\}+,
\end{aligned}
$$

where $x_{u o}=\int d r \Psi_{u}^{*} x \Psi_{o}$ and the subscripts $o$ and $u(i)$ stands for occupied and unoccupied state, respectively, and $U_{\mathrm{o}}$, $U_{i}$, and $U_{\mathrm{u}}$ are the corresponding Eigen-energies [43]. A typical minimum value of $U_{\mathrm{u}}-U_{\mathrm{o}}(E \mathrm{~g})$ of good FEs is $>3 \mathrm{eV}$, while a typical energy of $e x_{u o} E$ is $0.8 \mathrm{meV}$ 


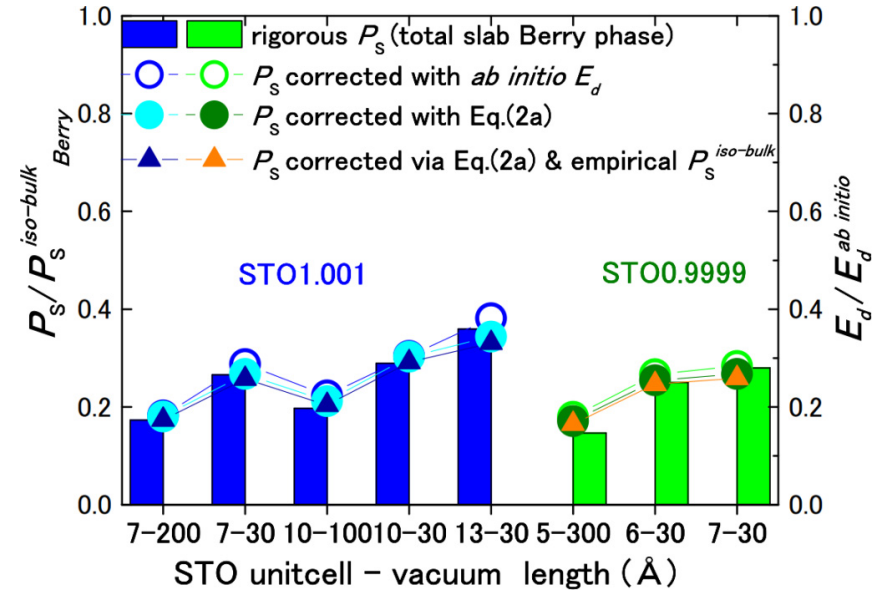

FIG. 6. Bars show the ratio of "Berry phase polarization $P_{\mathrm{S}}$ of a whole slab" to $P_{\mathrm{S}}^{\text {iso-bulk }}{ }_{\text {Berry }}\left[P_{\mathrm{S}}^{\text {iso-bulk }}{ }_{\text {Berry }}\right.$ : Berry phase polarization of extracted bulk (Fig. 1(f), Table I)]. Open circles show the ratios of " $P_{\mathrm{S}}$ calculated by Eq. (1) using $E_{d}^{a b i n i t i o " ~ t o ~} P_{\mathrm{S}}^{\text {iso-bulk }}$ Berry . Filled circles show the ratios of " $P_{\mathrm{S}}$ calculated by Eq. (1) using Eq. (2a)" to $P_{\mathrm{S}}^{\text {iso-bulk }}$ Berry . Numbers $n$ and $m$ in $n-m$ at the bottom represent the thicknesses of STO (in unit-cell) and vacuum (in $\AA$ ), respectively. Triangles show the ratios of $P_{\mathrm{S}}$ to $P_{\mathrm{S}}$ iso-bulk, where $P_{\mathrm{S}}$ is calculated by using Eq. (1), Eq. (2a), and $P_{\mathrm{S}}{ }^{\text {iso-bulk }}$ given by ion-position based empirical formula [12].

$\left(P_{\mathrm{el}}^{\text {Exra }} / 1 \mu \mathrm{C} / \mathrm{cm}^{2}\right)\left(E_{d} / 20 \mathrm{meV} / \AA\right) / N_{e}$, where $P_{\mathrm{el}}^{\text {Exra }}=P_{\mathrm{S}}-$ $P_{\mathrm{S}}{ }^{\text {iso-bulk }}$ and $N_{e}$ is the number of the contributing electrons per unit-cell. In case of $\mathrm{ABO}_{3}$ type FEs, the electrons per unit-cell contributing to the lowest $U_{\mathrm{u}}-U_{\mathrm{o}}$ are the $\mathrm{O}_{2 \mathrm{p}}$ valence electrons near the Fermi level $E_{\mathrm{F}}$, and $N_{e}$ is considered as 4 . Therefore, $e x_{u o} E \ll U_{\mathrm{u}}-U_{\mathrm{o}}$, and the third- and higher-order terms are unimportant. This suggests that $\varepsilon^{\mathrm{el}}$ can be approximated as a constant even for a high electric field with a good accuracy. Therefore, we use the linear static electron permittivity obtained in Fig. 5, while similar examinations show that the nonlinearity is not negligible for the permittivity of ions.

The possible dependence of $\varepsilon^{\mathrm{el}}$ on $u$ and, hence, $P_{\mathrm{S}}$ neglected, which is justified by Fig. 5 and the agreements of Eq. (2a) with $E_{d}^{a b}$ initio in Sec. IV; For example, $\varepsilon^{\text {el }}$ of cubic BTO differs from $\varepsilon^{\mathrm{el}}$ of strained BTO only by $3 \%$, where $P_{\mathrm{S}}$ 's of these BTO's by HSEsol are 0 and $49 \mu \mathrm{C} / \mathrm{cm}^{2}$, respectively [39]. Furthermore, Fig. 5 shows that $P_{\mathrm{S}}$ iso-bulk and, hence, $u$ of STO1.005 and STO.9999 do not affect $\varepsilon^{\mathrm{el}}$ 's.

The exchange correlation functionals change the $a b$ initio $\varepsilon^{\mathrm{el}}$ 's much more than the difference between STO and BTO; PBEsol yields the largest $\varepsilon^{\text {el }}$ 's, and HSEsol yields the smallest $\varepsilon^{\text {el }}$. This difference is consistent with Eq. (3), because PBEsol yields the smallest $E \mathrm{~g}$ and HSEsol yields the largest $E \mathrm{~g}$. The experimental $\varepsilon^{\mathrm{el}}$ 's are the closest to the $\varepsilon^{\mathrm{el}}$ 's calculated $a b$ initio with $\mathrm{PBE}+U$.

\section{ACCURACY OF THE METHOD}

In this section, the total polarizations are normal to the surfaces and interfaces, and $\cos \theta=1$.

FE/vacuum: All the eight kinds of STO/vacuum slabs labelled by the combination numbers in Fig. 6 exhibited the density of states (DOS) of insulators [Fig. 2(e)], and, therefore, Eq. (2a) with $P_{I}^{\text {iso-bulk }}=0$ and $\varepsilon_{l}^{\mathrm{el}}=1$ was used. $E_{d}$ ranges from 40 to $120 \mathrm{mV} / \AA$, increasing with $l_{V} / l_{f}$ and $P_{\mathrm{S}}$. $\varepsilon^{\mathrm{el}}=6.2$ in Eqs. (1) and (2a) was a static limit of the electron permittivity calculated $a b$ initio with PBEsol, because the $a b$ initio calculations of other properties of STO/vacuum slabs were performed with PBEsol.

In Fig. 6, the ratios of rigorously calculated Berry phase $P_{\mathrm{S}}$ of the whole slab to $P_{\mathrm{S}}{ }^{\text {iso-bulk }}$ are shown by bars, where $P_{\mathrm{S}}{ }^{\text {iso-bulk }}$ is the $a b$ initio spontaneous polarization $P_{\mathrm{S}}^{\text {iso-bulk }}$ of an extracted bulk unit-cell consisting of 5 atoms similar to Table I. The ratios of $P_{\mathrm{S}}$ to $P_{\mathrm{S}}^{\text {iso-bulk }}$ Berry are shown by open and filled circles, where the open circles correspond to $P_{\mathrm{S}}$ by Eq. (1) using ab initio $E_{d}\left(E_{d}^{a b \text { initio }}\right)$ and the filled circles correspond to $P_{\mathrm{S}}$ by Eq. (1) using Eq. (2a). Because the ion positions of all the unit-cells in each slab are exactly the same, $P_{\mathrm{S}}$ iso-bulk, $\mathrm{s}$ of all the unit-cells are exactly the same.

Both $P_{\mathrm{S}}$ using $E_{d}^{\text {abinitio }}$ and $P_{\mathrm{S}}$ using $E_{d}$ of Eq. (2a) agree excellently with rigorous ones. If one thinks that $P_{\mathrm{S}}$ iso-bulk is the total spontaneous polarization, which is conventional, the depolarization field $E_{d}^{\text {iso-bulk }}$ for $\mathrm{FE} /$ vacuum is $E_{d}^{\text {iso-bulk }}=-P_{\mathrm{S}}$ iso-bulk $/ \varepsilon_{0}\left(1+l_{f} / l_{I}\right)$. Therefore, $E_{d} / E_{d}^{\text {iso-bulk }}=P_{\mathrm{S}} / P_{\mathrm{S}}{ }^{\text {iso-bulk }}$, where $E_{d}=-P_{\mathrm{S}} / \varepsilon_{0}\left(1+l_{f} / l_{I}\right)$ is derived from Eqs. (1) and (2a) [30,44]. These ratios are shown in the right axis of Fig. 6. The appropriateness and importance of non-Born polarization $P_{\mathrm{el}}^{\mathrm{Exa}}$ are evident in the small ratio of $P_{\mathrm{S}} / P_{\mathrm{S}}{ }^{\text {iso-bulk }}$ and $E_{d} / E_{d}^{\text {iso-bulk }}$ as well as the excellent agreement of $P_{\mathrm{S}}=P_{\mathrm{S}}{ }^{\text {iso-bulk }}+P_{\mathrm{el}}^{\text {Exra }}$ with rigorously calculated $P_{\mathrm{S}}$. These agreements for the whole range of $E_{d}=40 \sim 120 \mathrm{meV}$ justify also the neglect of the nonlinearity of $\varepsilon^{\mathrm{el}}$ in Sec. IIID.

Additionally, $P_{\mathrm{S}}$ 's are calculated by Eqs. (1) and (2a) with $P_{\mathrm{S}}{ }^{\text {iso-bulk, }}$, given by $P_{\mathrm{S}}^{\text {iso-bulk }}=926 \Delta z_{\mathrm{Ti}-\mathrm{O} 2}-0.05\left(\mu \mathrm{C} / \mathrm{cm}^{2}\right)$ [12], where $\Delta z_{\mathrm{Ti}-\mathrm{O} 2}$ is the $z$ component of the distance between $\mathrm{Ti}$ and $\mathrm{O}$ in $\mathrm{TiO}_{2}$ plane $(z \| c$ axis) normalized by $c$-lattice constant of the unit-cell. The ratios of these $P_{\mathrm{S}}$ 's to $P_{\mathrm{S}}^{\text {iso-bulk }}$ Berry 's are shown in Fig. 6.

FE/insulator: All four BTO/STO slabs, labelled by the combination numbers in Fig. 7, exhibited DOS of states of insulators, and, hence, Eq. ( $2 \mathrm{a})$ is used. $E_{d}$ ranges from 15 to $23 \mathrm{mV} / \AA$, decreasing with $l_{f}$. The accuracy of Eqs. (1) and (2a) was tested by the comparison of the Berry phase $P_{\mathrm{S}}$ of a whole slab with $P_{\mathrm{S}}^{\text {slab }}{ }_{\text {average }} \equiv\left(l_{f} P_{\mathrm{S}}+l_{I} P_{I}\right) /\left(l_{f}+l_{I}\right)$, where $P_{\mathrm{S}}$ and $P_{I}$ are calculated by Eqs. (1) and (2a). This test was performed using the 7-unit-cell BTO/5-unit-cell STO $(\mathrm{PBE}+U)$, and the ratio was 1.000 .

The ratios of $P_{\mathrm{S}}$ to $P_{\mathrm{S}}{ }^{\text {iso-bulk }}$ are shown in Fig. 7(a), where $P_{\mathrm{S}}$ is calculated by both Eq. (1) using $E_{d}^{a b i n i t i o}$ and Eq. (1) using Eq. (2a). $P_{\mathrm{el}}^{\text {Exra }}$ changes $P_{\mathrm{S}}{ }^{\text {iso-bulk }}$ by $4 \%$ owing to small $E_{d}$. However, $P_{\mathrm{el}}^{\text {Exra }}$ changes $E_{d}$ 's substantially as seen Fig. 7(b). $E_{d}$ 's obtained through Eq. (2a) agree excellently

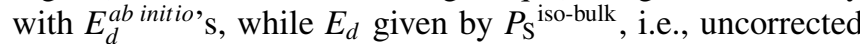
spontaneous polarization is $6 \sim 8$ times larger than $E_{d}^{a b}$ initio . These results prove the appropriateness and importance of $P_{\mathrm{el}}^{\text {Exra }}$.

To test the practical estimations, $E_{d}$ was calculated by Eq. (2a) using $P_{\mathrm{S}}$ iso-bulk's estimated by semi-empirical formulas $\quad P_{\mathrm{S}}{ }^{\text {iso-bulk }}(\mathrm{BTO})=977 \Delta z_{\mathrm{Ti}-\mathrm{O} 2}+0.04\left(\mu \mathrm{C} / \mathrm{cm}^{2}\right)$ and $P_{\mathrm{S}}{ }^{\text {iso-bulk }}(\mathrm{STO})=926 \Delta z_{\mathrm{Ti}-\mathrm{O} 2}-0.05\left(\mu \mathrm{C} / \mathrm{cm}^{2}\right) \quad$ [12]. The ratios of these $E_{d}$ 's to $E_{d}^{a b}$ initio's are shown as "by $\mathrm{TiO}_{2}$ " in Fig. 7(b). 


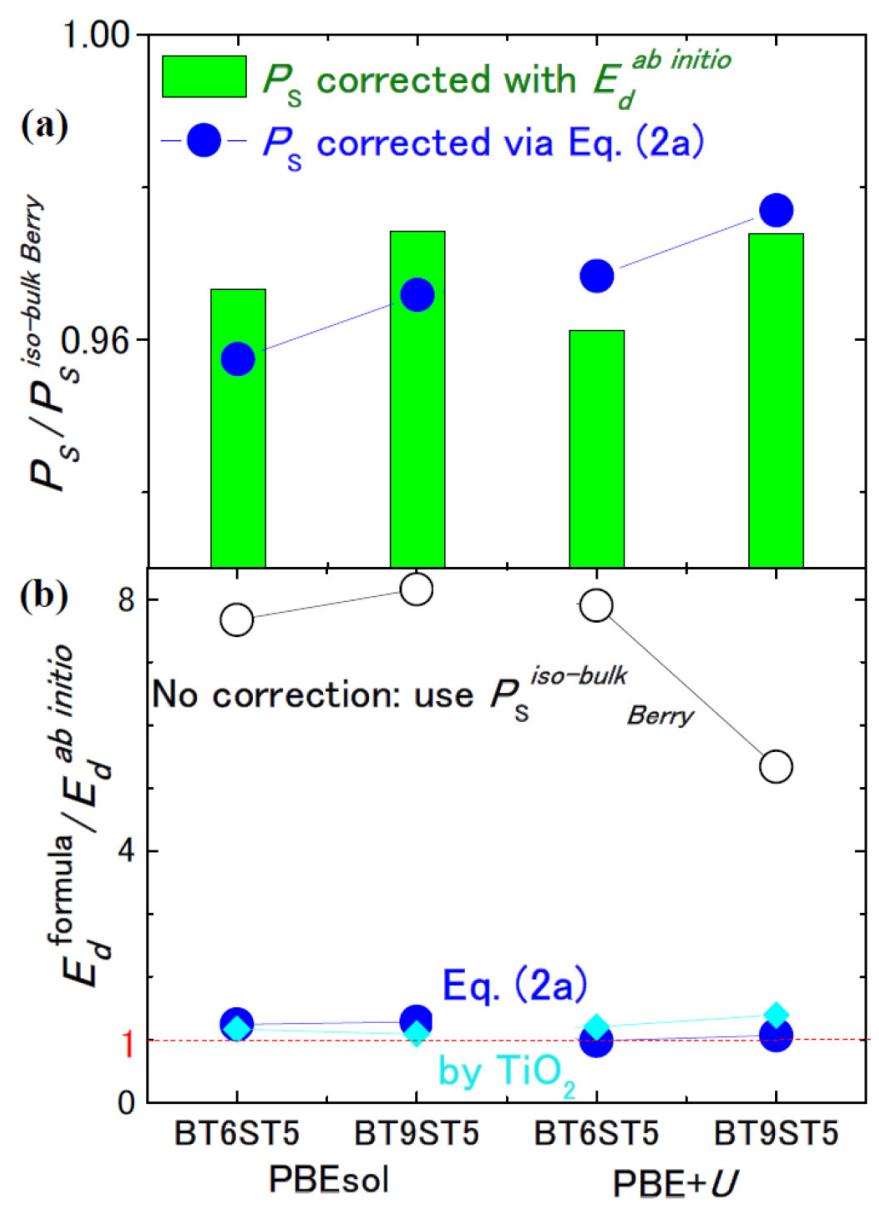

FIG. 7. (a) Bars show the ratio of " $P_{\mathrm{S}}$ calculated by Eq. (1)" using $E_{d}^{a b \text { initio }}$ to $P_{\mathrm{S}}^{\text {iso-bulk }}$ Berry. Filled circles show the ratio of " $P_{\mathrm{S}}$ by Eq. (1) using Eq. (2a)" to $P_{\mathrm{S}}^{\text {iso-bulk }}$ Berry . (b) Filled blue circles show the ratio of " $E_{d}$ calculated with $P_{\mathrm{S}}$ and $P_{I}$ (corrected by Eq. (2a))" to $E_{d}^{a b i n i t i o}$. Open black circles show the ratio of " $E_{d}$ calculated with $P_{\mathrm{S}}^{\text {iso-bulk }}$ Berry and $P_{I}^{\text {iso-bulk }}$ Berry (uncorrected)" to $E_{d}^{a b \text { initio. The supercell }}$ geometry BT $n \mathrm{ST} m$ at the bottom represents the number of unit-cells in BTO and STO. Light blue diamonds show the ratios of " $E_{d}$ by Eq. (2a)" to $E_{d}^{a b \text { initio }}$, where Eq. (2a) used $P_{\mathrm{S}}{ }^{\text {iso-bulk }}$ and $P_{I}^{\text {iso-bulk }}$ estimated by ion-position based empirical formula [12].

Additionally, the layer-by-layer $P_{\mathrm{S}}$ of each unit-cell was calculated by Eqs. (1) and (2b) in Fig. 4(b), where $\varepsilon_{j}^{\text {el }}$ 's by $\mathrm{PBE}+U$ were $5.36(\mathrm{BTO})$ and $5.22(\mathrm{STO})$ and $l_{j}$ 's were the distance between $\mathrm{Ba}$ or $\mathrm{Sr}$ atoms. For the layer-by-layer $P_{\mathrm{S}}$, $P_{j}^{\text {iso-bulk, }}$ sere estimated by the above semi-empirical formulas [12] using the layer-by-layer $\Delta z_{\mathrm{Ti}-\mathrm{O} 2}$ in Fig. 4(a) and agreed with $P_{\mathrm{S}}^{\text {iso-bulk }}$ Berry 's within $4 \%$ as seen in Fig. 4(b).

The layer-by-layer $P_{\mathrm{S}}{ }^{\text {iso-bulk }}$ varies substantially at the BTO-STO boundaries [Fig. 4(b)], yielding polarization charge $-\nabla \cdot P_{\mathrm{S}}{ }^{\text {iso-bulk }}$. The rescaled Born effective charges [23] yield the $P_{\mathrm{S}}$ variation similar to $P_{\mathrm{S}}$ iso-bulk, because of the uniform scaling. However, electrons free from ions, i.e., $P_{\mathrm{el}}^{\mathrm{Exra}}$ are expected to smoothen the profile and reduce the variations at the BTO/STO boundaries, because these electrons are considered to screen electric field from $-\nabla \cdot P_{\mathrm{S}}{ }^{\text {iso-bulk }}$. This expectation is realized in $P_{\mathrm{S}}$ in Fig. 4(b), proving the appropriateness and usefulness of the non-Born-polarization $P_{\mathrm{el}}^{\text {Exra }}$.

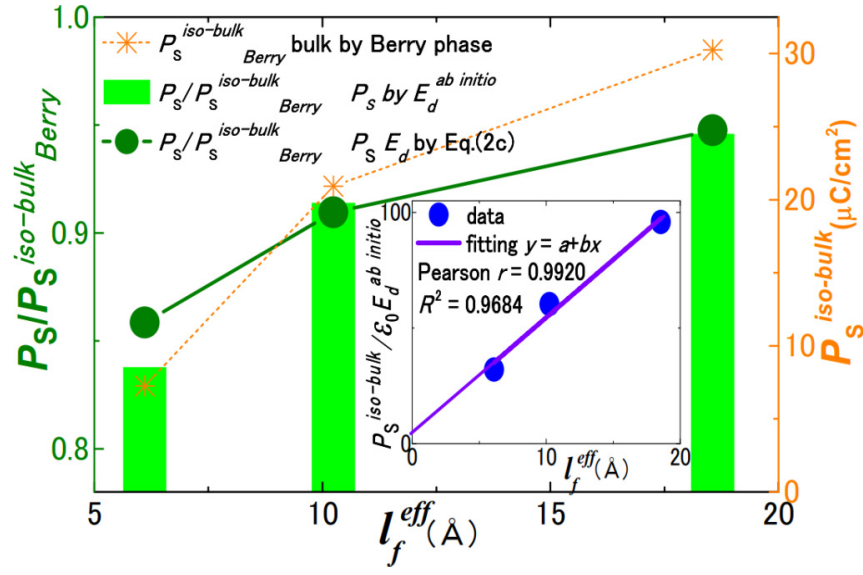

FIG. 8. Ratios of " $P_{\mathrm{S}}$ by Eq. (1)" and $E_{d}^{\text {ab initio }}$ to $P_{\mathrm{S}}^{\text {iso-bulk }}{ }_{\text {Berry }}$ shown by bars. Ratios of " $P_{\mathrm{S}}$ by Eqs. (1) and (2c)" to $P_{\mathrm{S}}^{\text {iso-bulk }}{ }_{\text {Berry }}$ are shown by filled dark-green circles. Asterisks show $P_{\mathrm{S}}^{\text {iso-bulk }}$ Berry . Inset: Estimation of $\varepsilon_{1} / 2 l_{1}$ by liner fitting to $P_{\mathrm{S}}^{\text {iso-bulk }}{ }_{\text {Berry }} / \varepsilon_{0} E_{d}$ vs. $l_{f}^{\text {eff }}$ plot.

Short-circuited capacitor: The rigorous Berry phase calculation of $P_{\mathrm{S}}$ of these slabs are not possible. Additionally, because the thickness of the screening layer in short-circuited capacitors is less than 1 unit-cell, the polarization of an extracted

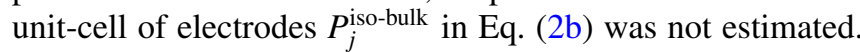
Therefore, we used $E_{d}=-P_{\mathrm{S}}{ }^{\text {iso-bulk }} / \varepsilon_{0}\left(\varepsilon^{\mathrm{el}}+\varepsilon_{1} l_{f} / 2 l_{1}\right)$ given by Eq. (2c), for which only the spontaneous polarization of an extracted bulk unit-cell of FE $P_{\mathrm{S}}{ }^{\text {iso-bulk }}$ was required. In Fig. 8, $P_{\mathrm{S}}$ iso-bulk decreases as $l_{f}^{\text {eff }}$ decreases, while $E_{d}$ ranges from 26 to $39 \mathrm{mV} / \AA$, reaching the maximum at $l_{f}^{\text {eff }} \approx 10 \AA$.

In the inset of Fig. $8, \varepsilon_{1} / 2 l_{1}$ was estimated as the inclination in $P_{\mathrm{S}}$ iso-bulk $/ \varepsilon_{0} E_{d}^{a b i n i t i o}$ vs. $l_{f}^{\text {eff }}$ plot and was $5.005 \AA^{-1}$, agreeing with the previous estimate [23]. $P_{\mathrm{S}}$ calculated by Eq. (1) with $E_{d}^{a b i n i t i o}$ is by $5 \% \sim 15 \%$ lower than $P_{\mathrm{S}}$ iso-bulk, showing the importance of $P_{\mathrm{el}}^{\text {Exra }}$. These $P_{\mathrm{S}}$ 's agree well with the $P_{\mathrm{S}}$ 's calculated by Eq. (1) with Eq. (2c) $\left[-P_{\mathrm{S}}{ }^{\text {iso-bulk }} / \varepsilon_{0}\left(\varepsilon^{\mathrm{el}}+\varepsilon_{1} l_{f} / 2 l_{1}\right)\right]$, which is performed only with ion positions of a representative unit-cell and $\varepsilon_{1} / 2 l_{1}$. Additionally, $E_{d}$ calculated by a macroscopic formula using $P_{\mathrm{S}}\left[E_{d}=-P_{\mathrm{S}} / \varepsilon_{0}\left(1+l_{f} / l_{I}\right)\right.$ derived from Eqs. (1) and (2a)] agreed with $E_{d}^{a b i n i t i o}$ better than those without correction, i.e., $P_{\mathrm{S}}=P_{\mathrm{S}}{ }^{\text {iso-bulk }}$.

\section{APPLICATION OF THE METHOD AND DOMAIN}

The preceding results are for the depolarization field $E_{d}$, but the present method is similarly applicable for external field $E_{\text {ext }}$. For theoretical studies of insulting FEs under high fields such as MD and ab initio calculations [3-5,7-10,19,24,25], non-Born polarization $P_{\mathrm{el}}^{\text {Exra }}$ is necessary. Here, insulting ideal FEs requires the absence of defects.

For experimental studies, the necessity of $P_{\mathrm{el}}^{\text {Exra }}$ depends on sample and time-scale, on which the magnitude of $E_{d}$ depends. However, in any case, $E_{d}$ should be lower than the conventional estimates. As an example of a long time-scale case, we discuss persisting vortexlike $P_{\mathrm{S}}$ patterns, which are reported experimentally and attributed conventionally to $E_{d}$ by the authors [3-5]. When these patterns are due to $E_{d}$ [3-5], $E_{d}$ at many locations should be high enough to change the 
original $P_{\mathrm{S}}$ almost entirely and exists persistently in FE. The persistence of such high field can only be possible, when FE is unusually insulating and, hence, exceptionally stoichiometric. For such cases, the correction $P_{\mathrm{el}}^{\text {Exra }}$, which reduces $E_{d}$ but was missing so far, are necessary.

On the contrary, when FE in experiments [3-5] is not unusually insulating, the correction $P_{\mathrm{el}}^{\text {Exa }}$ is unnecessary. In this case, however, $E_{d}$ cannot persist and, hence, cannot produce persisting vortexlike $P_{\mathrm{S}}$ patterns. That is, the primary origin of experimental vortexlike $P_{\mathrm{S}}$ patterns is not $E_{d}$, when $\mathrm{FE}$ is not unusually insulating.

A reference of insulativity of metal-oxide thin films is $\mathrm{LaAlO}_{3} / \mathrm{STO}$, which is exhaustively studied and exhibits defects and interdiffusion despite a state of art thin film growth technique $[7,45]$. The growth of stoichiometric metal-oxide thin film FE is more difficult than $\mathrm{LaAlO}_{3} / \mathrm{STO}$, because FEs contain highly volatile elements like $\mathrm{Pb}, \mathrm{Bi}, \mathrm{Li}, \mathrm{K}$, and $\mathrm{Sb}$. Actually, scanning TEM experiments of strain-induced FE-STO at $\mathrm{LaAlO}_{3} / \mathrm{STO}$ showed that $E_{d}$ was almost zero, or of order of impurity ionization energies [8]. Therefore, the contribution of $E_{d}$ to experimental vortexlike $P_{\mathrm{S}}$ patterns may be inessential, requiring other origins [26-28]. This inference may be consistent with the recent studies of Berezinskii-KosterlitzThouless phase in 2D-FEs [46].

One of the origins is strain, which is considered as the origin of $a / c\left(90^{\circ}\right)$ domains of tetragonal FEs [26-28]. Natural half flux-closure domains consisting of $a / c\left(90^{\circ}\right)$ domains were reported by Hooton and Merz in 1955 [28], and Gregg [26] concluded that all the reported vortexlike $P_{\mathrm{S}}$ patterns were flux-closure domains that can reduce both strain and $E_{d}$. Indeed, all the vortexlike $P_{\mathrm{S}}$ patterns observed by TEM were not found at free surfaces but at the interface of FEs [3-5] that could provide enormous strain. The disappearance of domains on a metallic ( $\mathrm{La}, \mathrm{Sr}) \mathrm{MnO}_{3}$ layer [5] can be explained also by the reduced film quality of FE layer due to the $(\mathrm{La}, \mathrm{Sr}) \mathrm{MnO}_{3}$ insertion and the decrease of strain. If these inferences are appropriate, vortexlike $P_{\mathrm{S}}$ patterns [3-5] can be nanometerscale descriptions of classical $a / c$ domains like Hooton and Merz that can reduce both strain and $E_{d}$ [28].

\section{SUMMARY}

For given ion positions or a unit-cell of insulating FEs and paraelectrics, the polarization $P_{\mathrm{S}}$ iso-bulk is estimated by standard methods such as ab initio calculations, Born effective charges, and empirical formula [2-5,7-10,11,12,15,19,24,25]. These estimations can be inadequate for the polarization under the depolarization field $E_{d}$, because $E_{d}$ is determined by global boundary conditions and, hence, is absent in the bulk unit-cell extracted from the entire structure, e.g., slab; A unit-cell extracted from the total structure possesses the ion positions that corresponds to $E_{d}$, but $E_{d}$ is absent in this unit-cell (Fig. 1, Table I). Consequently, unless electron polarization is completely associated with ion distortion $u$, a part of electron polarization, i.e., a part of atomic polarizability by $E_{d}$ $\left(P_{\mathrm{el}}^{\mathrm{Exa}}\right)$ is absent in these estimates, suggesting the inadequateness of polarization estimation based on local ion positions $P_{\mathrm{S}}$ iso-bulk $(\mathrm{Sec} . \mathrm{IV})$. Because $P_{\mathrm{el}}^{\text {Exra }}$ expresses the breakdown of the ion-position-polarization correspondence that is the basis of Born effective charges approaches (Appendix), we call $P_{\mathrm{el}}^{\mathrm{Exra}}$ non-Born effective charge polarization.

For the polarization under field, we postulated $P_{\mathrm{el}}^{\mathrm{Exra}}$ and proposed a method that calculated the total polarization $P_{\mathrm{S}}=$ $P_{\mathrm{S}}^{\text {iso-bulk }}+P_{\mathrm{el}}^{\text {Exra }}$ with $P_{\mathrm{el}}^{\text {Extra }}=\left(\varepsilon_{\mathrm{el}}^{\text {Extra }}-1\right) \varepsilon_{0} E$ or Eq. (1). This method worked, even when ab initio Berry phase calculation was not possible, and the correctness of the postulate and the high accuracy were demonstrated in Sec. IV, especially $P_{\mathrm{S}}$ and $E_{d}$ of STO/vacuum and BTO/STO. In addition, the global consistency of $P_{\mathrm{S}}{ }^{\text {iso-bulk }}$ yielded formulas that calculated both $P_{\mathrm{S}}$ and $E_{d}$, using static electron permittivity, layer-thicknesses, and $P_{\mathrm{S}}{ }^{\text {iso-bulk }}$, which could also estimate $3 \mathrm{D} P_{\mathrm{S}}$ and $E_{d}$ in unitcell-scale. Here, the unit-cell-scale layer-by-layer $P_{\mathrm{S}}$ could be also performed by algebra instead of $a b$ initio Wannier approaches [23]. Because $P_{\mathrm{S}}{ }^{\text {iso-bulk }}$ is the conventionally estimated polarization, i.e., Born polarization, for which experimental ion positions can be used $[11,12]$, these formulas enable the experimental estimation of $P_{\mathrm{S}}$. The present method revealed a layer-by-layer $P_{\mathrm{S}}$ variation markedly flatter than that of conventional estimate $P_{\mathrm{S}}{ }^{\text {iso-bulk }}$, explaining successfully a small $E_{d}$ and the absence of metallic layer formation by $E_{d}$ $[17,18]$. The reduction of $E_{d}$ is expected also for the situations of vortexlike domains. Because the order parameter of FE in GL theories is a total polarization [15], the incomplete entanglement of ion and electron polarization shown here implies limitation of GL framework of FE under high field.

\section{ACKNOWLEDGMENTS}

The discussions with P. Blöchel and the support from JSPS KAKENHI Grant No. JP19K21853 are acknowledged.

\section{APPENDIX}

Extracted unit-cell: $P_{\mathrm{S}}$ iso-bulk is the polarization of an extracted or copied unit-cell in which ion positions are exactly the same as those in the slab [Fig. 1(e)] and, hence, the ion distortion $u$ due to $E_{d}$ is fully contained [Fig. 1(f)] (Sec. I). This copied unit-cell can be called as a bulk with frozen ions as Table I shows; The ion positions and lattice of the copied unit-cell for $P_{\mathrm{S}}{ }^{\text {iso-bulk }}$ is the same as those of a unit-cell in the slab. In case of layer-by-layer estimation of $P_{\mathrm{S}}{ }^{\text {iso-bulk }}$, the extractions or copies similar to Table I are performed for all the unit-cells in the slab.

Born effective charges: Practical Born effective charges are not accurate as Berry phase calculations [20], because they are intended to estimate many different materials. However, Born effective charges can agree perfectly with Berry phase calculations, by incorporating $u$-dependence of $Z^{*}$, and other effects such as defects specific to the target FE. This possibility is confirmed by the perfect agreement of the curves expressed by a single $u$ with Berry phase calculations in Fig. 9(a), where the polynomial for $P_{\mathrm{S}}$ includes the $u$-dependence of $Z^{*}$. The difference in $P_{\mathrm{S}}$ by the two method is invisible, whereas the difference in $P_{\mathrm{S}}$ by Berry phase calculations with PBEsol and TPSS [47] is evident. Additionally, Berry phase $P_{\mathrm{S}}$ of a bulk BTO having the experimental ion positions and lattice constants at $298 \mathrm{~K}$ ranged between 23 and $24 \mu \mathrm{C} / \mathrm{cm}^{2}$ [39]. These results suggest that $a b$ initio calculations are also approximations, although they are excellent. The quasilinearity 

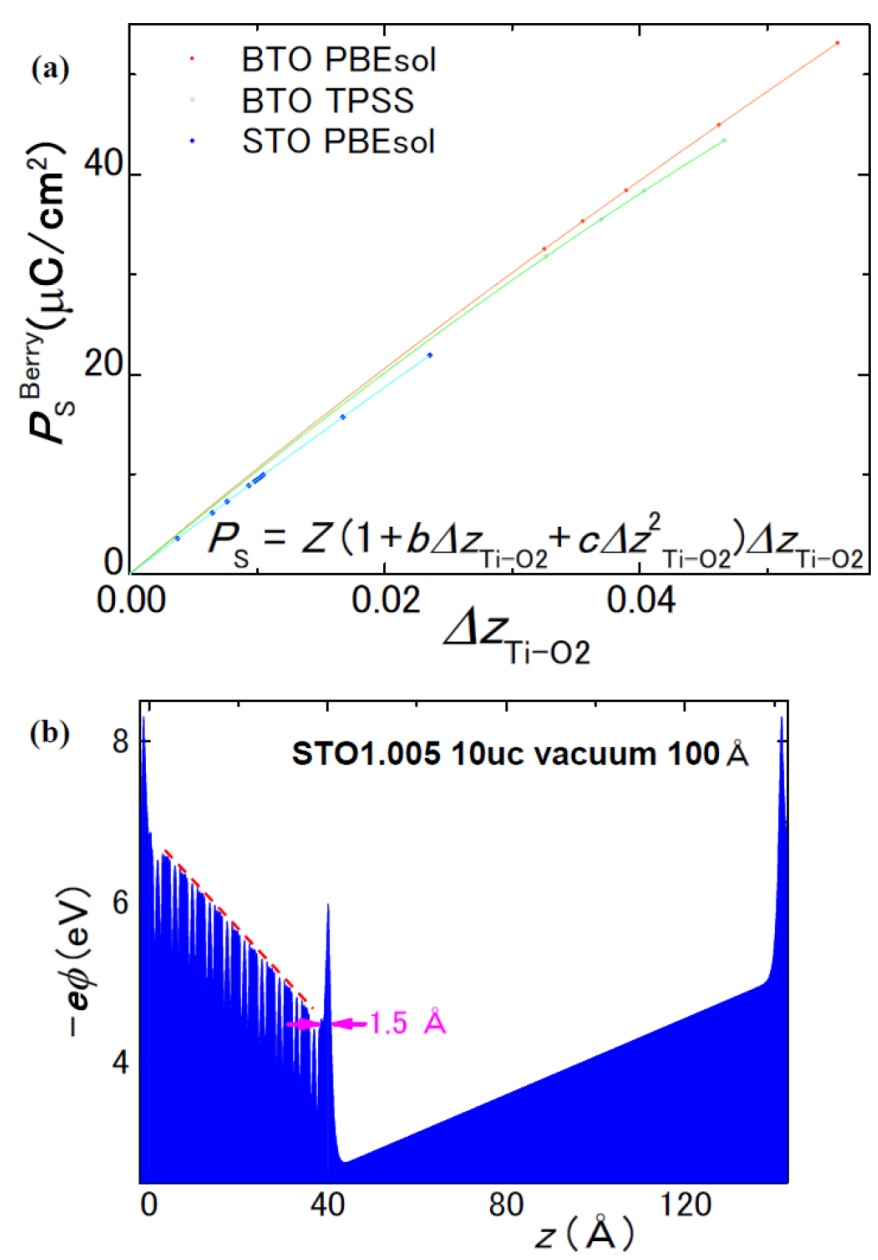

FIG. 9. (a) Relationship between Ti-O $\mathrm{O}_{2}$ distortion $u\left(\Delta z_{\mathrm{Ti}-\mathrm{O} 2}\right)$ and Berry phase $P_{\mathrm{S}}$ of BTO and STO calculated $a b$ initio using specific exchange correlations extracted from Ref. [12], indicating that exact $P_{\mathrm{S}}$ can be obtained by Born effective charges. The curves by the polynomial in (a) are plotted by solid lines, where $Z, b$, and $c$ are constants. (b) Example of the thickness of the surface layer of $\mathrm{FE} /$ vacuum and the estimation of $E_{d}^{a b}$ initio from the highest planar averaged $\phi$ by the $a b$ initio calculation.

of the curves in Fig. 9 supports the practical accuracy of the standard Born effective charges $\Sigma Z^{*} u$ ( $Z^{*}$ : constant).

Therefore, both ideal Born effective charges and ideal $a b$ initio calculations should yield the same $P_{\mathrm{S}}$ iso-bulk, which is exact. Hence, because the physics or the spirit of Born effective charges is clear and describes the situation of $P_{\mathrm{S}}$ iso-bulk , we call
$P_{\mathrm{S}}{ }^{\text {iso-bulk }}$ Born polarization. Accordingly, the polarization not included in $P_{\mathrm{S}}^{\text {iso-bulk }}$, i.e., $P_{\mathrm{el}}^{\text {Exra }}$ is called as non-Born effective charge polarization.

STO1.005, STO.9999: To achieve the insulativity of FE used in Berry phase calculations of $P_{\mathrm{S}}$ of a whole slab, STO1.005 and STO.9999 were chosen (Sec. II). For freestanding FEs and HH-TT domains, $E_{d}=-P_{\mathrm{S}}{ }^{\text {iso-bulk }} / \varepsilon_{0} \varepsilon^{\mathrm{el}}$ by Eq. (2a), which yields $\phi_{\max }=l_{f} P_{\mathrm{S}}{ }^{\text {iso-bulk }} / \varepsilon_{0} \varepsilon^{\mathrm{el}}\left(\phi_{\max }\right.$ : maximum potential difference). Therefore, $l_{f} P_{\mathrm{S}}{ }^{\text {iso-bulk }} \varepsilon_{0} \varepsilon^{\mathrm{el}}<E g / e$ is required for insulativity [30]. This requires a very small $P_{\mathrm{S}}{ }^{\text {iso-bulk }}$, e.g., $3 \mu \mathrm{C} / \mathrm{cm}^{2}$ for $l_{f}$ sufficiently long for the estimation of $E_{d}$ by the method in Fig. 2(d). This is the reason for choosing STO1.005 and STO.9999. For example, even a 2-unit-cell-thick ( $\sim 8$ ) BTO in vacuum was metallic, when ion positions were fixed at those of a bulk BTO [48]. In case of STO1.005, 10-unit-cell-STO with $l_{\mathrm{V}}=100 \AA$ is marginally insulating, while 16-unit-cell-STO1.005 with $l_{\mathrm{V}}=30 \AA$ is metallic. In case of STO.9999, the critical $l_{f}$ is reduced as in Fig. 2(e), owing to the increase of $P_{\mathrm{S}}$ iso-bulk

Additionally, the $a b$ initio calculations by us and in literature (all ions relaxed) of 1D-FE/vacuum and 1D-HH-TT domains $[10,16,19]$ show only two alternates: FE with metallic surface or paraelectric insulator, as predicted analytically $[6,17]$. Therefore, STO/vacuum was not relaxed to retain FE; Otherwise, it became paraelectric with $E_{d}=0$.

No dipole correction: The dipole correction is used to imitate the experimental surfaces, for which $l_{\mathrm{V}}=\infty$. The present target is not the FE surfaces $\left(l_{\mathrm{V}}=\infty\right)$ but FE/vacuum superlattices, because they are sufficient for the examinations of Eqs. (1)-(2c). Hence, the dipole correction was not applied.

Here, the change of $P_{\mathrm{S}}$ by the interactions between top and bottom surface is not a problem for the examinations of Eqs. (1)-(2c) by the comparison with $a b$ initio results. These examinations were accurate, because we used $a b$ initio $P_{\mathrm{S}}$ iso-bulk, which contained the effect of the above interaction, in Eqs. (1)-(2c) and, hence, that effect was self-consistently included in both $a b$ initio results and Eqs. (1)-(2c).

Surface layer and oblique case: Figure 9(b) shows that the thickness of the surface layer deviating macroscopic $E_{d}$, e.g., Eq. (2a) is $\sim 1.5 \AA$ in FE/vacuum. For $\theta \neq 0$, Eq. (2b) changes to

$E_{d k}=-\frac{\sum_{j=1}^{N}\left\{\left(P_{k} \cos \theta_{k}-P_{j} \cos \theta_{j}\right) l_{j} / \varepsilon_{j}^{e l}\right\}}{\varepsilon_{k}^{e l} \varepsilon_{0} \sum_{j=1}^{N} l_{j} / \varepsilon_{j}^{e l}} \quad(k=1 \sim N)$,

where $\theta_{j}$ is the angle of the polarization $P_{j}$ to the $1 \mathrm{D}$ direction.
[1] C. Kittel, Rev. Mod. Phys. 21, 541 (1949).

[2] P. Chen, M. P. Cosgriff, Q. Zhang, S. J. Callori, B. W. Adams, E. M. Dufresne, M. Dawber, and P. G. Evans, Phys. Rev. Lett. 110, 047601 (2013)

[3] A. K. Yadav et al., Nature 530, 198 (2016).

[4] C.-L. Jia, K. W. Urban, M. Alexe, D. Hesse, and I. Vrejoiu, Science 331, 1420 (2011).
[5] C. T. Nelson et al., Nano Lett. 11, 828 (2011).

[6] Y. Watanabe, J. Appl. Phys. 83, 2179 (1998); Erratum: 84, 3428 (1998).

[7] C.-P. Su, A. K. Singh, T. C. Wu, M. C. Chen, Y. C. Lai, W. L. Lee, G. Y. Guo, and M. W. Chu, Phys. Rev. Materials 3, 075003 (2019).

[8] P. W. Lee et al., Nat. Commun. 7, 12773 (2016). 
[9] S. Kouser, T. Nishimatsu, and U. V. Waghmare, Phys. Rev. B 88, 064102 (2013); T. Nishimatsu, U. V. Waghmare, Y. Kawazoe, and D. Vanderbilt, ibid. 78, 104104 (2008).

[10] N. Sai, C. J. Fennie, and A. A. Demkov, Phys. Rev. Lett. 102, 107601 (2009).

[11] S. C. Abrahams, S. K. Kurtz, and P. B. Jamieson, Phys. Rev. 172, 551 (1968).

[12] Y. Watanabe, Comput. Mater. Sci. 158, 315 (2019).

[13] Ph. Ghosez, X. Gonze, Ph. Lambin, and J.-P. Michenaud, Phys. Rev. B 51, 6765(R) (1995); X. Gonze and C. Lee, ibid. 55, 10355 (1997); M. Posternak, R. Resta, and A. Baldereschi, ibid. 50, 8911 (1994); J. D. Axe and W. A. Harrison, Electronic Structure and the Properties of Solids (Dover, New York, 1980).

[14] O. Diéguez, K. M. Rabe, and D. Vanderbilt, Phys. Rev. B 72, 144101 (2005).

[15] A. F. Devonshire, Philos. Mag. 40, 1040 (1949); especially p. 1056; 42, 1065 (1951); especially p. 1067.

[16] S. Liu and R. E. Cohen, J. Phys.: Condens. Matter 29, 244003 (2017).

[17] Y. Watanabe, Phys. Rev. B 57, 789 (1998); Y. Watanabe and D. Sawamura, Jpn. J. Appl. Phys. 36, 6162 (1997); Y. Watanabe, Ferroelectr. 333, 57 (2006); Y. Watanabe and A. Masuda, Integr. Ferroelectr. 27, 51 (1999).

[18] Y. Watanabe, M. Okano, and A. Masuda, Phys. Rev. Lett. 86, 332 (2001)

[19] J. Sifuna, P. G.-Fernández, G. S. Manyali, G. Amolo, and J. Junquera, Phys. Rev. B 101, 174114 (2020).

[20] C. Menéndez, D. Chu, and C. Cazorla, npj Comput. Mater. 6, 76 (2020).

[21] J. Mitroy, M. S. Safronova and C. W Clark, and M. Atoji, J. Chem. Phys. 25, 174 (1956).

[22] X. Wang and D. Vanderbilt, Phys. Rev. B 75, 115116 (2007).

[23] M. Stengel, P. Aguado-Puente, N. A. Spaldin, and J. Junquera, Phys. Rev. B 83, 235112 (2011); Born effective charge rescaling Eq. (33) or Eq. (A7) is described in the Appendix.

[24] I. I. Naumov, L. Bellaiche, and H. Fu, Nature 432, 737 (2004).

[25] Y.-H. Shin, I. Grinberg, I-W. Chen, and A. M. Rappe, Nature 449, 881 (2007).

[26] J. M. Gregg, Ferroelectr. 433, 74 (2012).

[27] S. K. Streiffer et al., J. Appl. Phys. 83, 2742 (1998).

[28] J. A. Hooton and W. J. Merz, Phys. Rev. 98, 409 (1955).

[29] Y. Watanabe, Phys. Rev. B 99, 064107 (2019).

[30] Y. Watanabe (unpublished).
[31] G. Kresse and J. Hafner, Phys. Rev. B 47, 558 (1993); G. Kresse and J. Furthmüller, Comput. Mater. Sci. 6, 15 (1996); Phys. Rev. B 54, 11169 (1996); G. Kresse and D. Joubert, ibid. 59, 1758 (1999); M. Hutchinson and M. Widom, Comput. Phys. Commun. 183, 1422 (2012); M. Hacene, A. A. Sedrakian, X. Rozanska, D. Klahr, T. Guignon, and P. F. Lessard, J. Comput. Chem. 33, 2581 (2012).

[32] P. E. Blöchl, Phys. Rev. B 50, 17953 (1994).

[33] J. P. Perdew, A. Ruzsinszky, G. I. Csonka, O. A. Vydrov, G. E. Scuseria, L. A. Constantin, X. Zhou, and K. Burke, Phys. Rev. Lett. 100, 136406 (2008).

[34] H. J. Monkhorst and J. D. Pack, Phys. Rev. B 13, 5188 (1976).

[35] R. Resta, Rev. Mod. Phys. 66, 899 (1994); R. D. King-Smith and D. Vanderbilt, Phys. Rev. B 47, 1651 (1993).

[36] J. P. Perdew, K. Burke, and M. Ernzerhof, Phys. Rev. Lett. 77, 3865 (1996).

[37] A. I. Liechtenstein, V. I. Anisimov, and J. Zaanen, Phys. Rev. B 52, R5467 (1995).

[38] J. Heyd, G. E. Scuseria, M. Ernzerhof, J. Chem. Phys. 118, 8207 (2003); L. Schimka, J. Harl, and G. Kresse, ibid. 134, 024116 (2011).

[39] Y. Watanabe, J. Chem. Phys. 148, 194702 (2018).

[40] 5. N. Bickel, G. Schmidt, K. Heinz, and K. Müller, Phys. Rev. Lett. 62, 2009 (1989).

[41] R. R. Mehta, B. D. Silverman, and J. T. Jacobs, J. Appl. Phys. 44, 3379 (1973).

[42] M. Cardona, Phys. Rev. 140, A651 (1963); W. L. Bond and A. R. Johnston, J. Appl. Phys. 42, 3501 (1971).

[43] J. J. Sakurai, Title (Addison Wesley, Redwood, 1985), Chap. 5.1, p. 296; L. I. Shiff, Quantum Mechanics, 3rd ed. (McGraw Hill, New York, 1968), Chap. 8, Sec. 33, p. 264; A. S. Kompaneyets and J. M. Ziman, Principles of the Theory of Solids, 2nd ed. (Cambridge University Press, Cambridge, UK, 1972), Chap. V, Sec. 5.6.

[44] Y. Watanabe, Ferroelectr. 461, 38 (2014).

[45] S. A. Pauli et al., Phys. Rev. Lett. 106, 036101 (2011); Y. Li, X. Wei, and J. Yu, J. Appl. Phys. 127, 205302 (2020).

[46] C. Xu, Y. Nahas, S. Prokhorenko, H. Xiang, and L. Bellaiche, Phys. Rev. B 101, 241402(R) (2020); J. W. Villanova, P. Kumar, and S. Barraza-Lopez, ibid. 101, 184101 (2020).

[47] J. Tao, J. P. Perdew, V. N. Staroverov, and G. E. Scuseria, Phys. Rev. Lett. 91, 146401 (2003).

[48] Y. Watanabe, Ferroelectr. 556, 29 (2020). 